\title{
EL USO DE PLATOS TRAMPA Y RED ENTOMOLÓGICA EN LA CAPTURA DE ABEJAS NATIVAS EN EL ESTADO DE NUEVO LEÓN, MÉXICO
}

\author{
LILIANA RAMÍREZ FREIRE, ${ }^{1}$ GLAFIRO ALANÍS FLORES, ${ }^{1}$ RICARDO AYALA \\ BARAJAS ${ }^{2}$, CARLOS VELAZCO MACÍAS ${ }^{3}$ y SUSANA FAVELA LARA ${ }^{1}$ \\ ${ }^{1}$ Facultad de Ciencias Biológicas, Universidad Autónoma de Nuevo León, 66451 San Nicolás de los \\ Garza, Nuevo León, México. ²Estación de Biología Chamela (Sede Colima) Instituto de Biología, \\ UNAM. ${ }^{3}$ Parques y Vida Silvestre. Av. Eugenio Garza Sada 6604, Col. Lagos del Bosque, 64890 \\ Monterrey, Nuevo León, México. Correspondencia: <biolily@gmail.com>
}

Ramírez Freire, L., Alanís Flores, G., Ayala Barajas, R., Velazco Macías, C. y Favela Lara, S. 2014. El uso de platos trampa y red entomológica en la captura de abejas nativas en el estado de Nuevo León, México. Acta Zoológica Mexicana (n.s.), 30(3): 508-538.

RESUMEN. Se realizó un estudio faunístico de abejas nativas en el estado de Nuevo León utilizando los métodos de platos trampa y red entomológica. Se recolectaron 3,952 ejemplares pertenecientes a cinco familias, 47 géneros y 191 especies que al sumar aquellas encontradas en literatura y bases de datos de colecciones revisadas el número se elevó a 317 especies. Se capturaron 73 especies únicamente en platos trampa, 68 exclusivamente con la red entomológica y 48 coincidiendo en ambos métodos. Se reportan por primera vez para el estado 7 géneros y 32 especies. En los platos trampa se colectó una mayor abundancia y riqueza de especies; mientras que la mayor diversidad se observó con el método de la red entomológica. De los platos trampa, el color amarillo capturó la mayor abundancia, riqueza y diversidad de abejas siendo la familia Halictidae la mejor representada. Durante el muestreo con red entomológica se identificaron 21 familias de plantas visitadas por abejas, entre las que destacan por la abundancia de éstas Scrophulariaceae, Asteraceae y Fabaceae; mientras que de la que se obtuvo mayor diversidad fue de Asteraceae. Se discute sobre el número de especies recolectadas y los resultados de la curva de acumulación, así como de los métodos de colecta empleados.

Palabras clave: Apoidea, abejas, métodos de captura, inventario faunístico, Nuevo León.

Ramírez Freire, L., Alanís Flores, G., Ayala Barajas, R., Velazco Macías, C. \& Favela Lara, S. 2014. Using pan traps and netting to collect native bees in Nuevo León state, Mexico. Acta Zoológica Mexicana (n.s.), 30(3): 508-538.

ABSTRACT. A wildlife study was carried out on native bees in the state of Nuevo Leon, northeast Mexico; color bowl traps and netting were used as sampling technique. A total of 3,952 individuals were collected, in five families, 47 genera and 191 species, also, adding those species recorded on literature and existing databases the species number raised to 317. A total of 73 species were collected by color bowl traps and 68 species were collected using entomological net, 48 species were collected using both

Recibido: 16/10/2013; aceptado: 29/04/2014 
methods. Seven genera and 32 species are reported for the first time for the state of Nuevo Leon. Higher abundance and species richness were recorded on color bowl traps, and higher diversity was recorded on entomological net. Yellow bowl traps captured the highest bee abundance, diversity and richness being Halictidae the best represented family. During entomological net sampling a total of 21 plant families were identified as visited by bees, plant families with highest bee abundance were Scrophulariaceae, Asteraceae and Fabaceae, highest bee diversity was recorded on Asteraceae. Discussion about number of species collected and accumulation curve are presented also on collection methods.

Key words: Apoidea, bees, bee collecting, faunal inventory, Nuevo León.

\section{INTRODUCCIÓN}

El fenómeno de disminución en la producción agrícola a causa de la pérdida de abejas a nivel mundial (Allen-Wardell et al. 1998, Kevan \& Phillips 2001), ha sido la pauta para evaluar la situación actual de estos insectos en muchos países y México no es la excepción (Quesada 2013); se crea entonces la necesidad de conocer las condiciones en las que se encuentra la fauna del país. Sin embargo, respecto a la superfamilia Apoidea aún falta mucho por investigar ya que la zona más estudiada se encuentra en la porción centro y sur del país (Ramírez-Freire 2008); por ende, de una gran extensión del territorio nacional se cuenta solamente con algunos muestreos esporádicos realizados principalmente por extranjeros (Ayala et al. 1996), situación que se refleja en la baja representatividad de abejas en las colecciones nacionales. Por tal motivo es necesaria la realización de inventarios faunísticos que permitan conocer la biodiversidad y para lograrlo es determinante definir un tipo de muestreo acorde con el grupo biológico a estudiar.

Los métodos más comunes para el muestreo de abejas se pueden clasificar en activos y pasivos (Potts et al. 2005). Dentro de los métodos activos se encuentran la observación visual directa o mediante cámaras de video, el uso de red entomológica a través del muestreo en transectos lineales o directamente en plantas en floración, así como el uso de aspiradores (Kearns \& Inouye 1993, Potts et al. 2005, Droege 2009). Entre los muestreos pasivos están la trampa Malaise, trampas ventana, platos trampa, trampas de cebo, trampas pegajosas (Kearns \& Inouye 1993, Dafni et al. 2005, Droege 2009), nidos trampa (Oliveira et al. 2012), etc.; la elección del método a aplicar dependerá del tipo de estudio que se pretenda realizar o el tipo de abejas que se desee colectar.

Desde la década pasada, el uso de platos trampa (“pan traps” o "bowl traps”) ha destacado, ya que se ha comprobado que es un método eficiente, imparcial y con un mayor costo-beneficio, en comparación con otros (Droege 2009, Droege et al. 2009). Asimismo se ha demostrado que es el mejor método en zonas áridas, debido a que es simple y eficiente para la captura de un mayor número de individuos; por otra parte, Grundel et al. (2011) señalan que la utilización de red entomológica como un método adicional de colecta ayuda a complementar los inventarios biológicos ya que especies poco comunes pueden ser registradas en uno u otro método. 
En México se han realizado diferentes estudios faunísticos como el de GodínezGarcía (1991), Roubik et al. (1991), Estrada (1992), Hinojosa-Díaz (1996), Ayala (1999), Vergara \& Ayala (2002), Hinojosa-Díaz (2003), Godínez-García et al. (2004), Ruiz et al. (2010), entre otros; sin embargo, la mayoría fueron llevados a cabo en estados del centro y sur empleando únicamente la red entomológica como método de captura. Se reconocen para el país 1,800 especies y subespecies de abejas agrupadas en 144 géneros y seis familias, siendo las de mayor riqueza Apidae (73 géneros y 597 especies) y Andrenidae (11 géneros y 532 especies). No obstante, a pesar del incremento en el número de publicaciones sobre este grupo, aún existen áreas del territorio nacional que no han sido estudiadas; además, el 56 \% de los géneros conocidos para México no han sido revisados taxonómicamente, por lo cual es necesario continuar con la recolecta de ejemplares para registrar el mayor número de especies de abejas mexicanas (Ayala et al. 1996).

De acuerdo a los registros existentes en la literatura (McGinley 1986, Ayala et al. 1996, Ayala 1999, Ramírez-Freire 2008) y con base en los ejemplares de abejas nativas depositados en colecciones entomológicas de México (UNIBIO 2011) y Estados Unidos (a través de Ascher \& Pickering 2010) disponibles en línea, existen en Nuevo León un total de 58 géneros y 157 especies. Sin embargo, el vacío de información que se presenta en el noreste de México constituye una razón de mayor importancia para la realización de un inventario faunístico de abejas; tomando en cuenta esta información el objetivo de la investigación consiste en comparar dos métodos de captura de abejas nativas en el estado de Nuevo León y de una manera colateral contribuir al conocimiento de la biodiversidad de este grupo.

\section{MATERIAL Y MÉTODOS}

Área de estudio. Se reúne la información recabada en 36 sitios, distribuidos en 20 municipios del estado de Nuevo León (Fig. 1), el cual se localiza al noreste de la República Mexicana, entre los $23^{\circ} 10^{\prime} 27^{\prime \prime}$ y $27^{\circ} 46^{\prime} 06^{\prime}$ ' de latitud norte y $98^{\circ} 26^{\prime} 24^{\prime}$ ' y $101^{\circ} 13^{\prime} 55^{\prime}$ ' de longitud oeste, el cual se ubica dentro de la gran zona árida del desierto Chihuahuense; sin embargo, la presencia de cadenas montañosas y la cercanía con el Golfo de México, propicia la existencia de bosques y matorrales altos (INEGI 2005). En el estado predomina el clima tipo B que es cálido y seco (INEGI, 1986); presenta diferentes tipos de vegetación que han sido tratados en Miranda \& Hernández (1963), Rojas (1965) y Rzedowski (1978) no obstante, prevalecen los matorrales (Alanís 1996).

Muestreo. El trabajo de campo fue realizado entre septiembre de 2008 y mayo de 2010. Para el muestreo se utilizaron los siguientes dos métodos de captura.

1. Platos trampa. Se elaboraron utilizando 120 recipientes de plástico de boca ancha de $150 \mathrm{ml}$ de capacidad de diferentes colores, 30 de ellos de color blanco; los restantes, divididos en números iguales, fueron teñidos con pintura en aerosol fluo- 


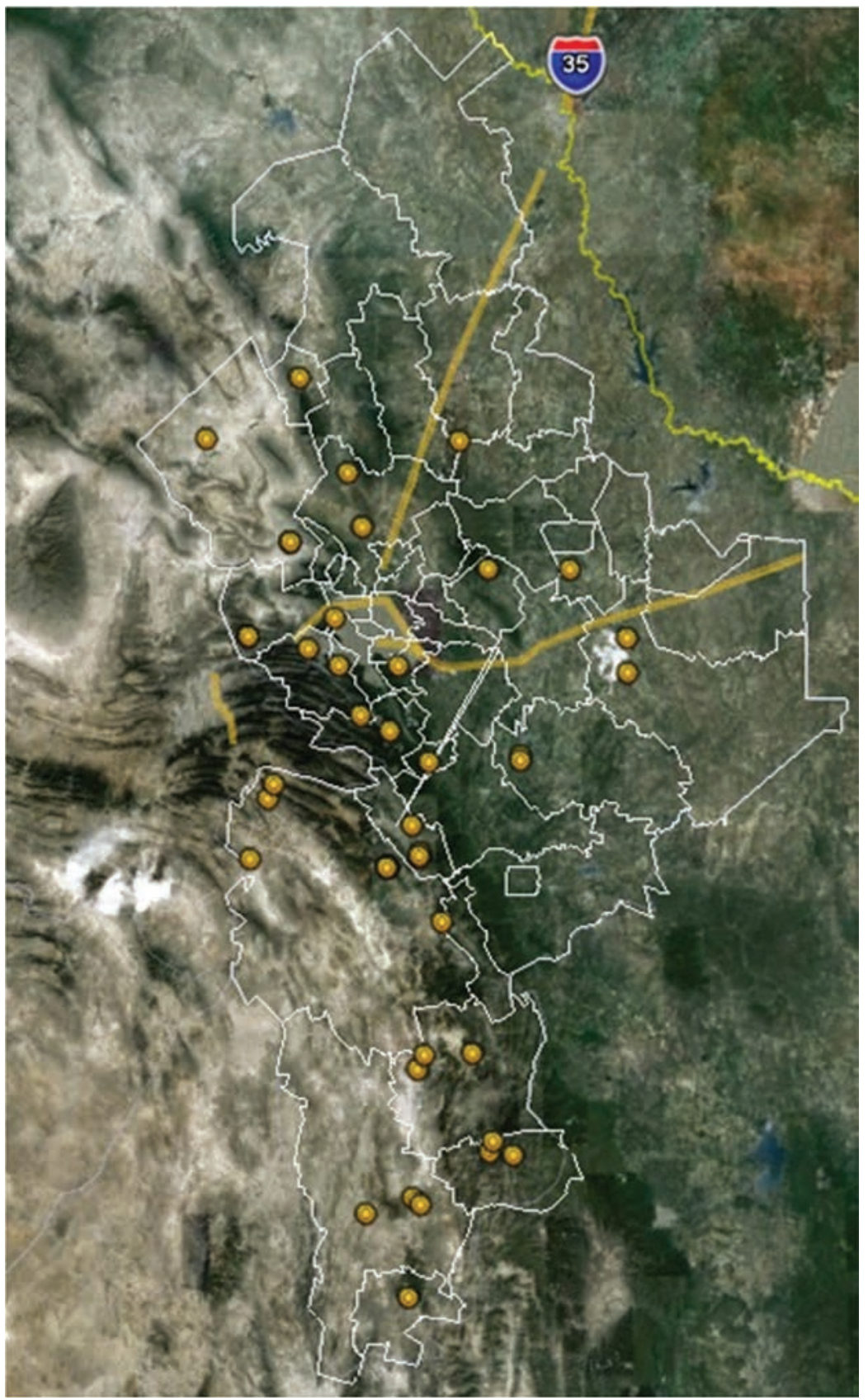

Figura 1. Localización de los sitios de muestreo para la colecta de abejas nativas en el estado de Nuevo León, México. 
rescente en los siguientes colores: rosa, amarillo y azul. Los platos fueron distribuidos en campo agregándoles una solución jabonosa (100 ml de agua y aproximadamente 1/2 cucharada de detergente líquido comercial para trastes) y colocados regularmente de 8:00 a 18:00 horas en zonas abiertas, a una distancia aproximada entre recipientes de $3 \mathrm{~m}$ e intercalando los colores. Las abejas atrapadas fueron extraídas de las trampas usando un tamiz para eliminar la solución jabonosa y procurando no mezclar organismos atrapados entre los distintos colores, luego se depositaron en frascos con alcohol etílico al 70\%. En el laboratorio, los ejemplares fueron lavados y secados según el método sugerido por Droege (2009); posteriormente fueron montados en seco, etiquetados y almacenados para su identificación taxonómica.

2. Red entomológica. Los muestreos fueron aleatorios y dirigidos hacia áreas con plantas silvestres en floración, empleando una red entomológica de golpeo; las muestras fueron tomadas durante media hora a diferentes intervalos de tiempo, entre las 9:00 y 16:00 horas. Se procuró que los sitios de muestreo estuvieran a una distancia aproximada de al menos un kilómetro de la ubicación de los platos trampa. Las abejas fueron sacrificadas en cámara letal usando acetato de etilo como ingrediente activo; posteriormente, se transportaron en viales y se montaron usando alfileres entomológicos. Además se tomaron muestras botánicas de las plantas sobre las que se realizó la colecta, las cuales fueron preparadas para su posterior identificación.

Las abejas fueron determinadas con las claves taxonómicas de Arduser (2009), Ascher \& Pickering (2010), Hurd (1955), Hurd \& Michener (1955), Labougle (1990), Michener et al. (1994), Michener (2007), Mitchell (1960 y 1962) y Roberts (1972); una parte fue identificada con ayuda del Dr. John L. Neff del Central Texas Melittological Institute. Los ejemplares fueron depositados en la Colección Entomológica de la Facultad de Ciencias Biológicas de la Universidad Autónoma de Nuevo León.

Análisis de datos. La cuantificación de especies se hizo mediante el valor de Riqueza Específica (S), y para estimar el número de especies se realizó una curva de acumulación, para lo cual se utilizó el programa EstimateS 8.2.0 (Colwel 2009). Para evaluar la calidad del muestreo y realizar la curva de acumulación, se utilizó la ecuación de Clench sugerida por Jiménez \& Hortal (2003). Para obtener la gráfica de la curva se empleó el software Statistica 10 (StatSoft 2012).

Dado que se están considerando la riqueza y abundancia como indicativos de eficiencia del tipo de muestreo, se procedió a evaluar esta información mediante índices de diversidad, ya que utilizan estas variables para su estimación. Para ambos métodos de muestreo los datos se sometieron a los índices de Margalef (DMg), Simpson (Ds) y Shannon $\left(\mathrm{H}^{\prime}\right)$ y para hacer comparaciones entre ellos se realizó una prueba T-Student para muestras independientes con un alfa de 0.05. Para los resultados del método de platos trampa se realizó un ANOVA de un factor con un alfa de 0.05 con el objetivo de hacer comparaciones entre los colores empleados, a los cuales también se les calculó el Î́ndice de Jaccard para contrastar la similitud de especies entre ellos; 
asimismo este índice se aplicó a los resultados obtenidos con la red entomológica pero sólo para las familias de plantas que presentaron la mayor riqueza de abejas.

\section{RESULTADOS}

Se recolectaron 3,952 ejemplares, con una Riqueza Específica (S) de 191 especies de abejas en 47 géneros y cinco familias. En total 32 especies y siete géneros se reportan por primera vez para el estado de Nuevo León; de éstos 17 fueron colectados con red, siete en los platos trampa y ocho en ambos métodos (Cuadro 1). Apidae presentó el mayor número de especies, mientras que la menor cantidad se registró en la familia Colletidae (Fig. 2).

En cuanto a la riqueza estimada, en la Figura 3 se aprecia la línea obtenida del modelo de la curva de acumulación de especies y en ésta el valor obtenido del factor $\mathrm{R}^{2}$ que fue de 0.997, el cual indica que los datos obtenidos del muestreo se ajustan bien al modelo. El valor de riqueza estimado ( $a$ : 10.981/ b: 0.031) arrojó una proyección de 354 especies.

De los ejemplares capturados, se obtuvieron 73 especies de los platos trampa, 68 exclusivamente con la red entomológica y 48 coincidieron en ambos métodos. En el

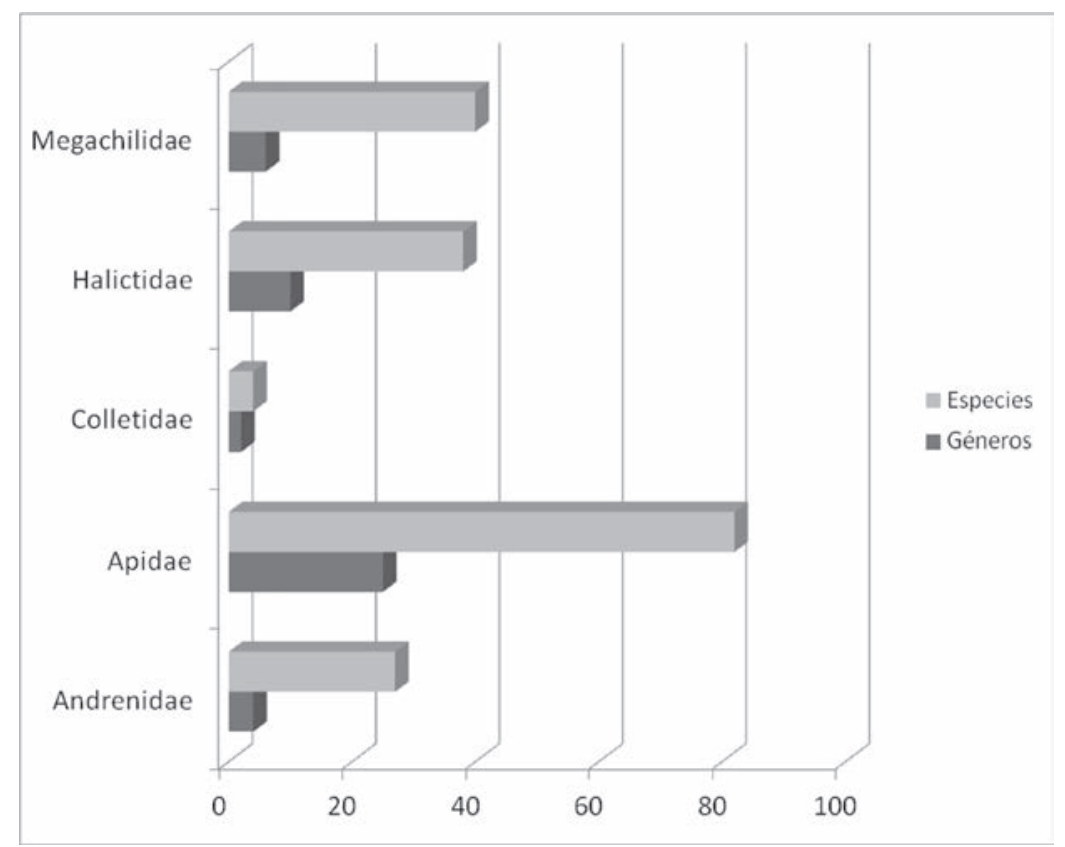

Figura 2. Riqueza de géneros y especies de las familias de abejas nativas colectadas mediante el uso de platos trampa y red entomológica en el estado de Nuevo León, México. 


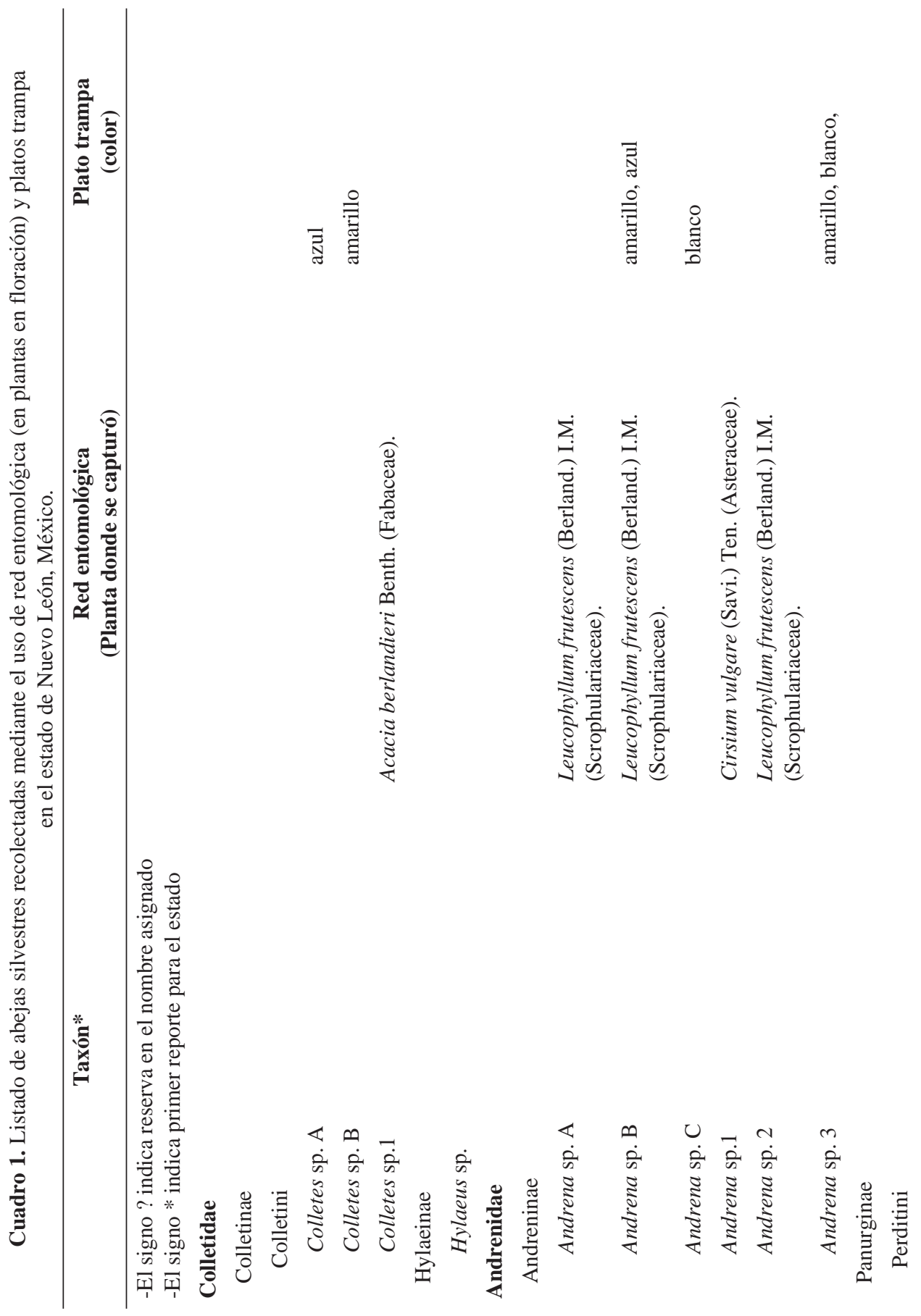




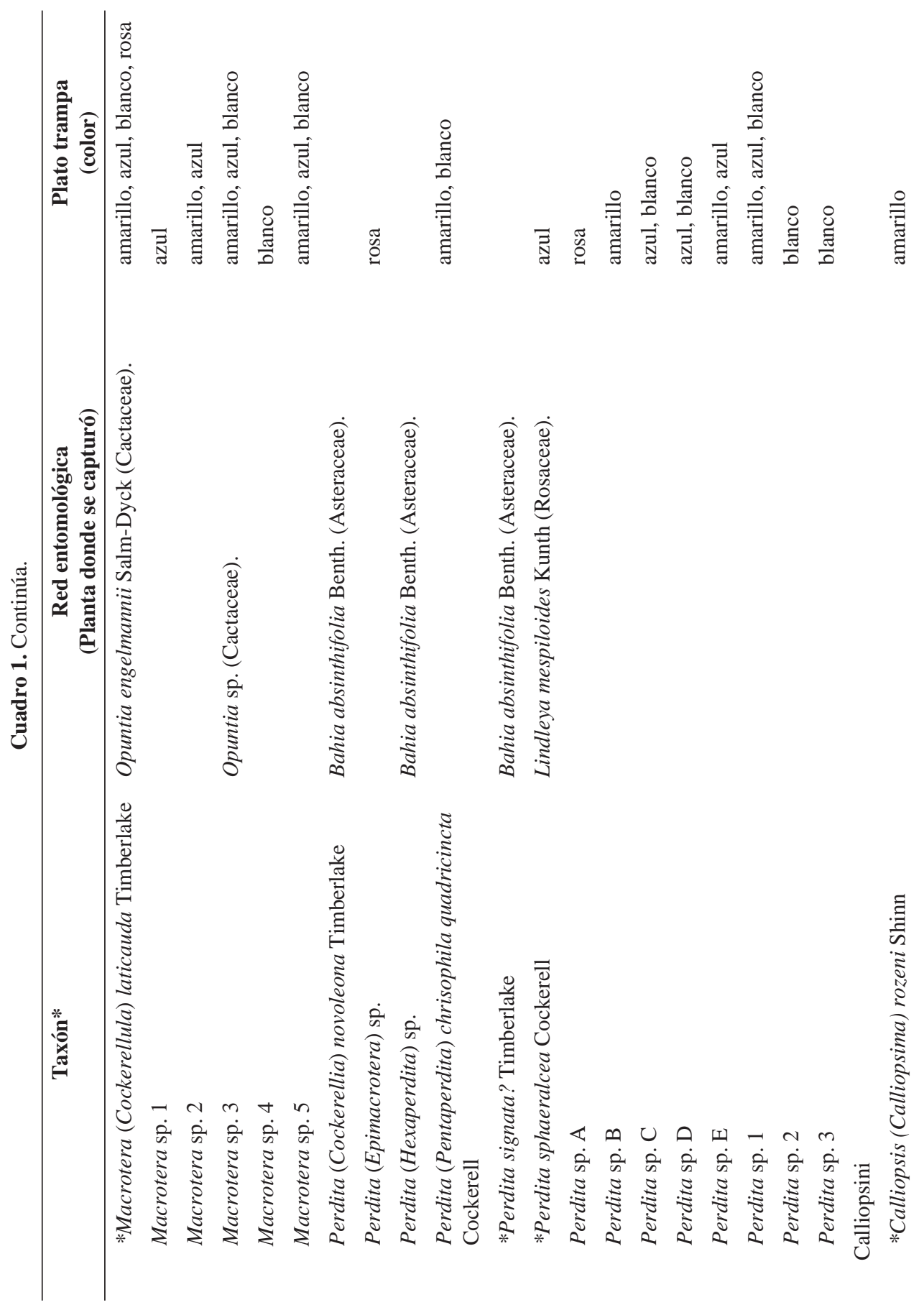




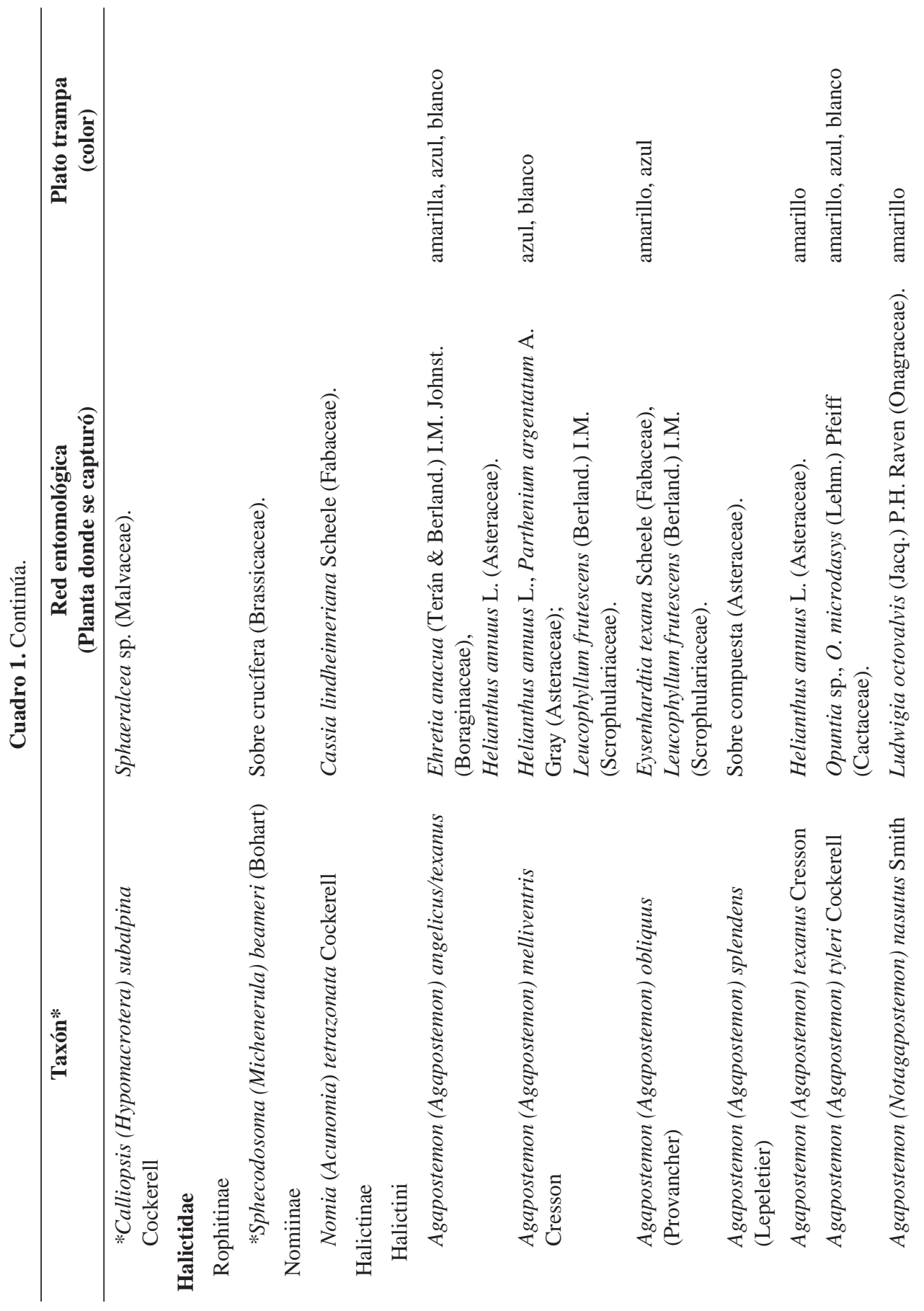




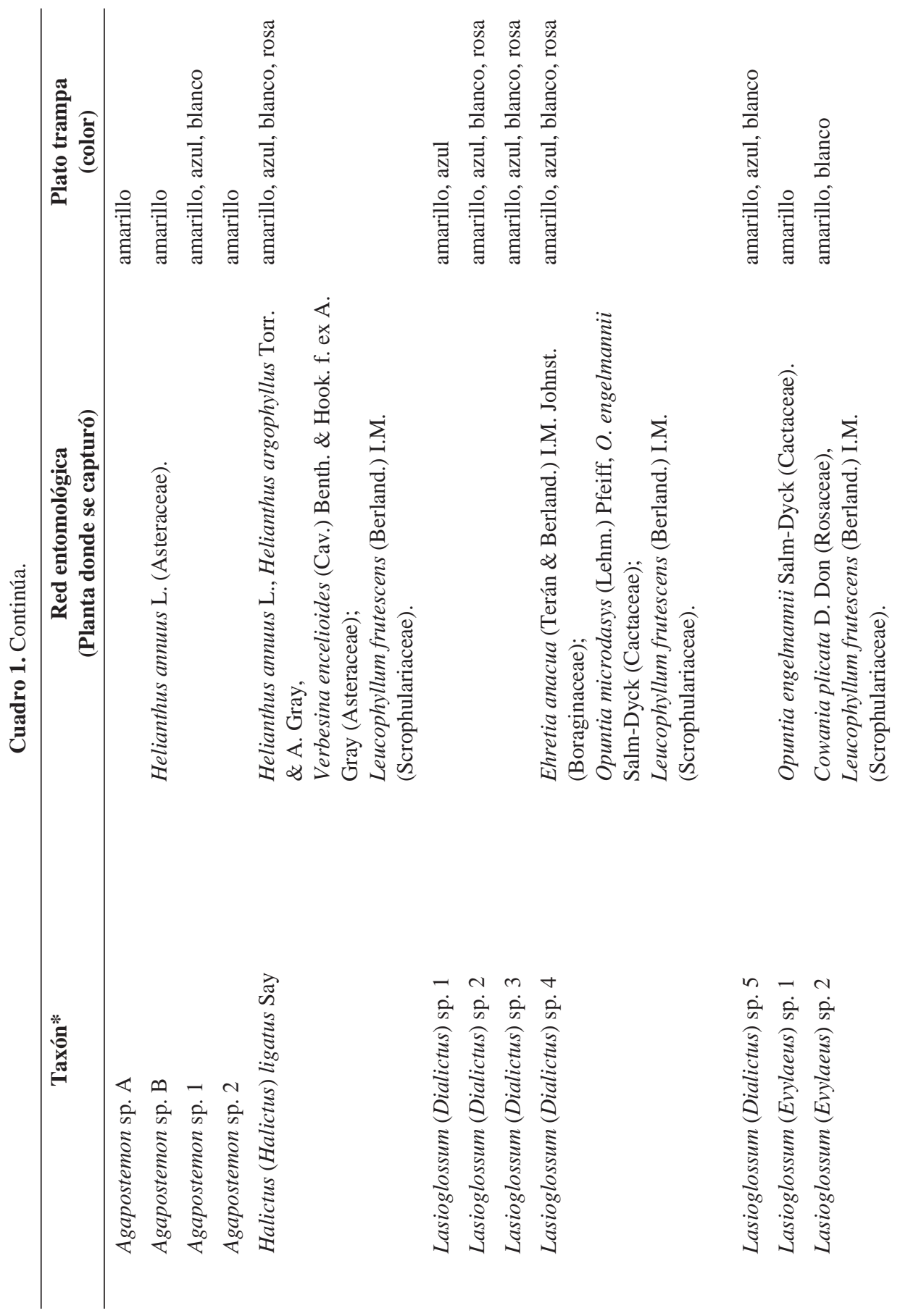




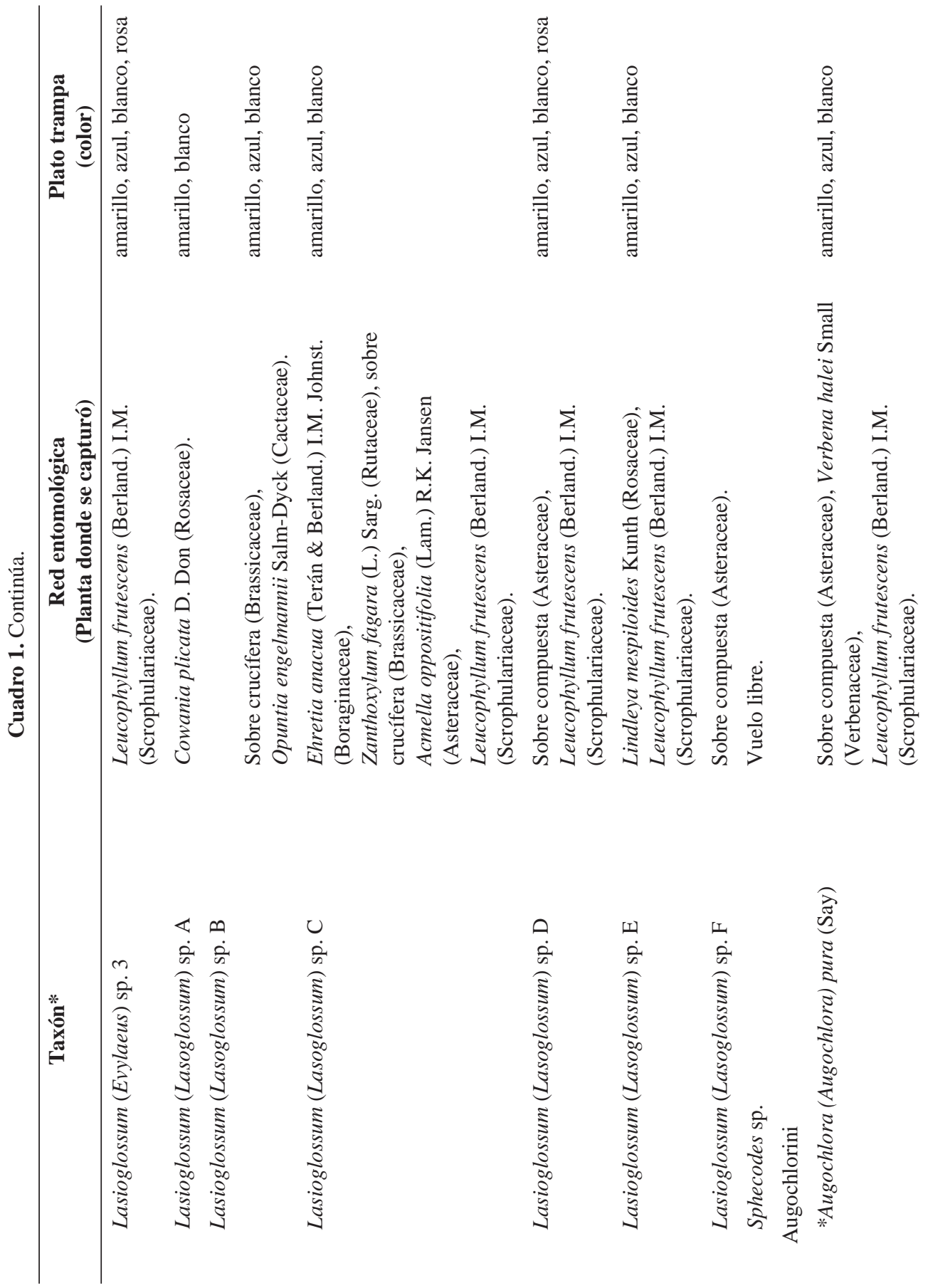




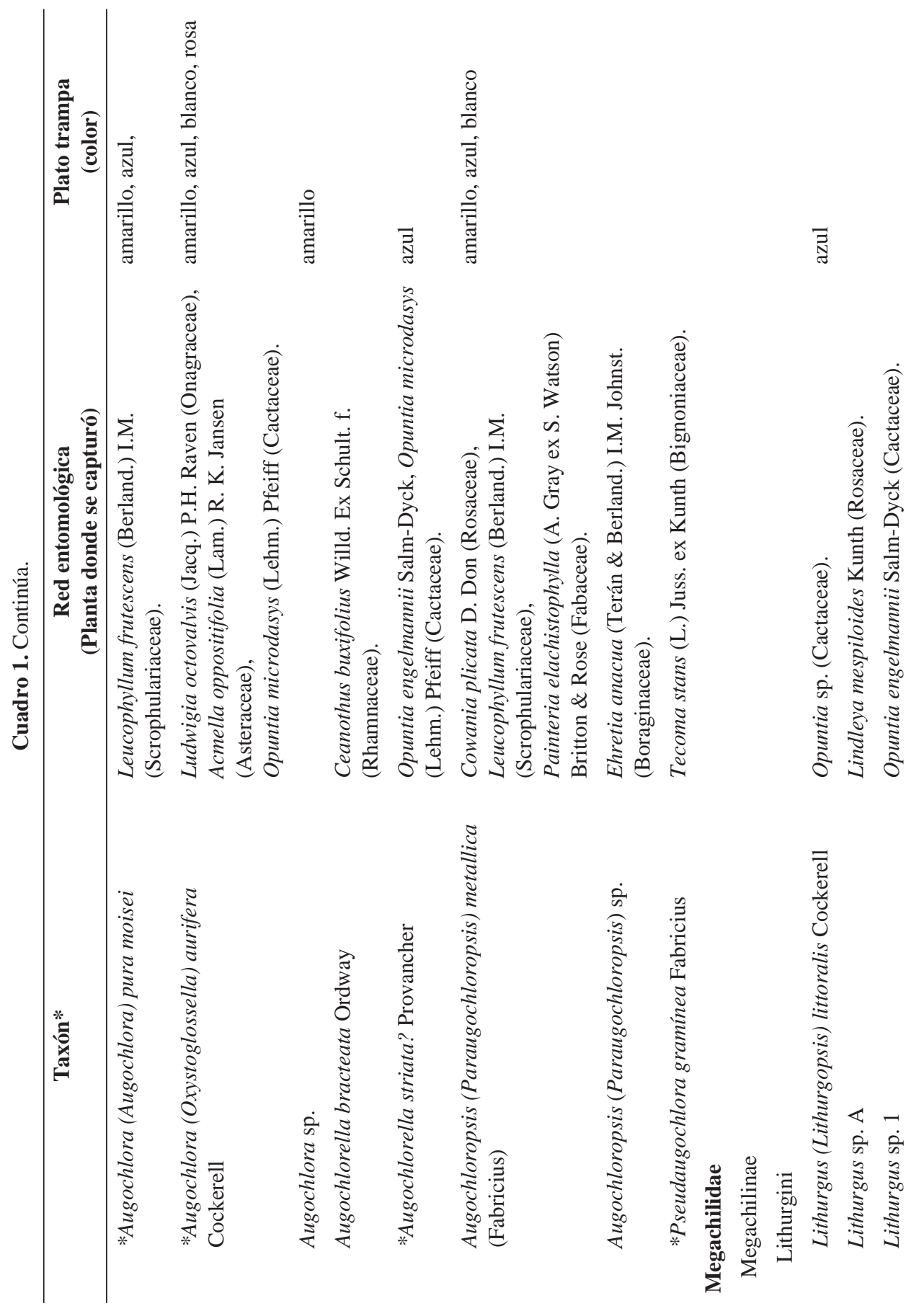




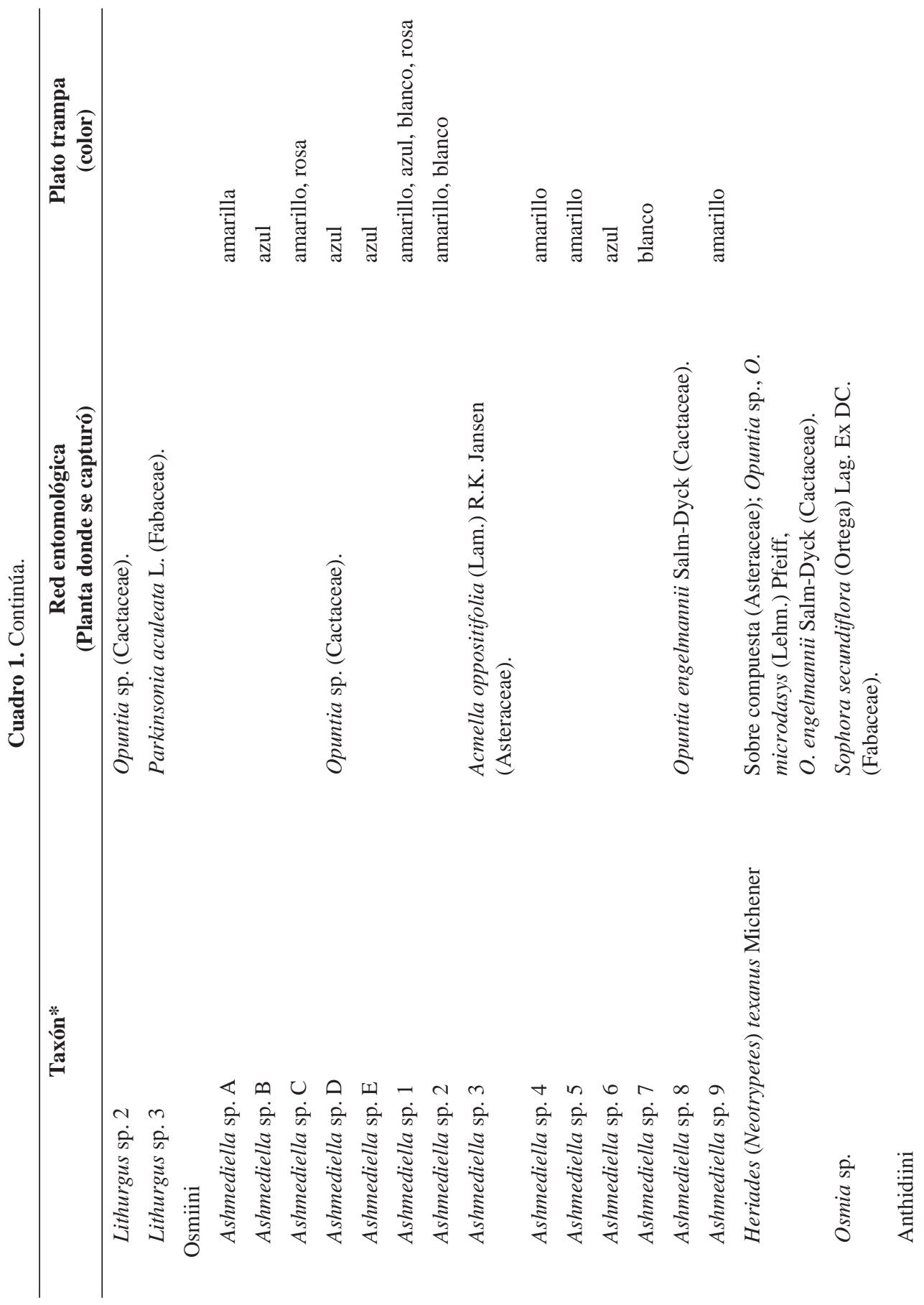




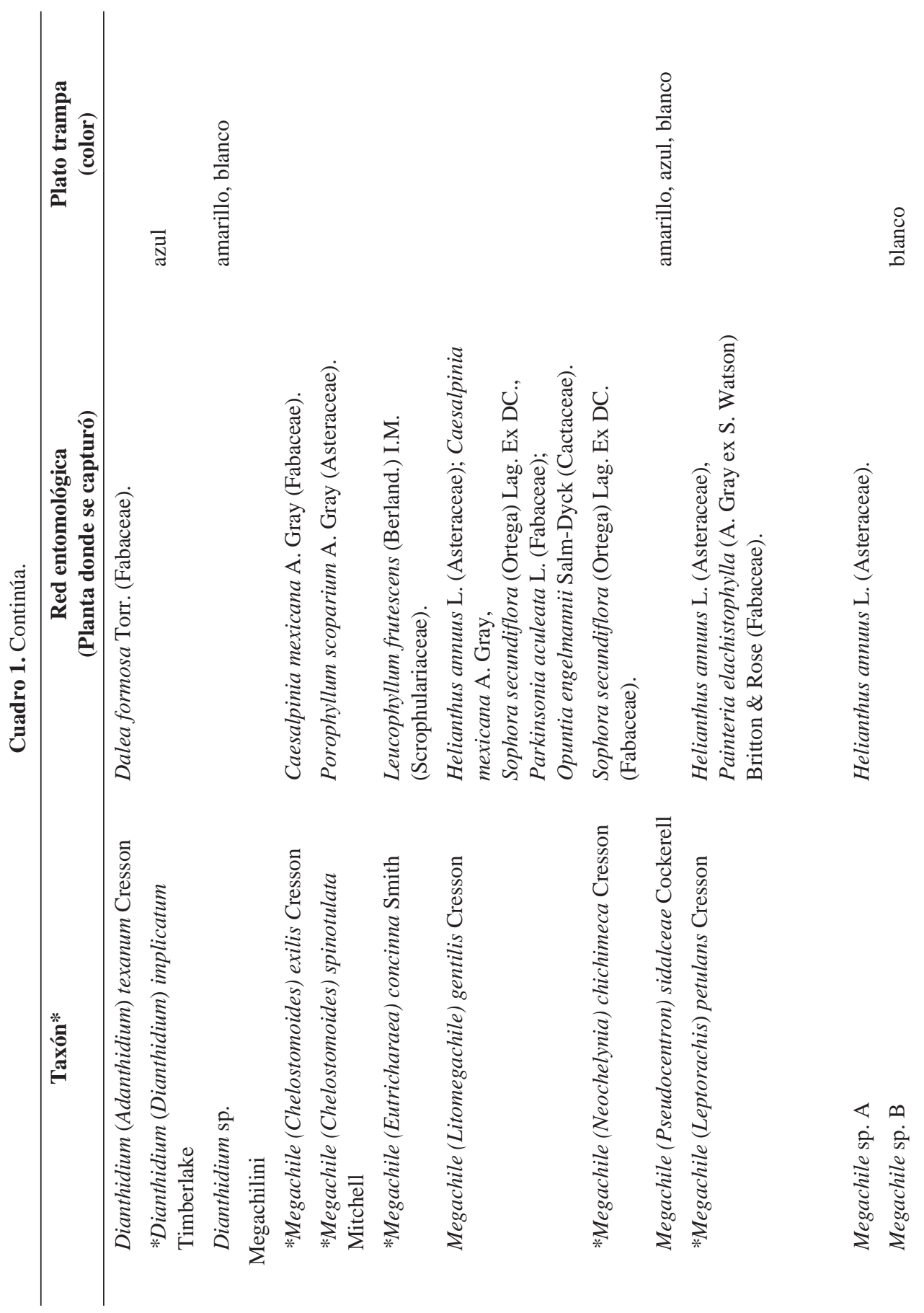


Ramírez Freire et al.: Métodos de captura para abejas nativas en Nuevo León

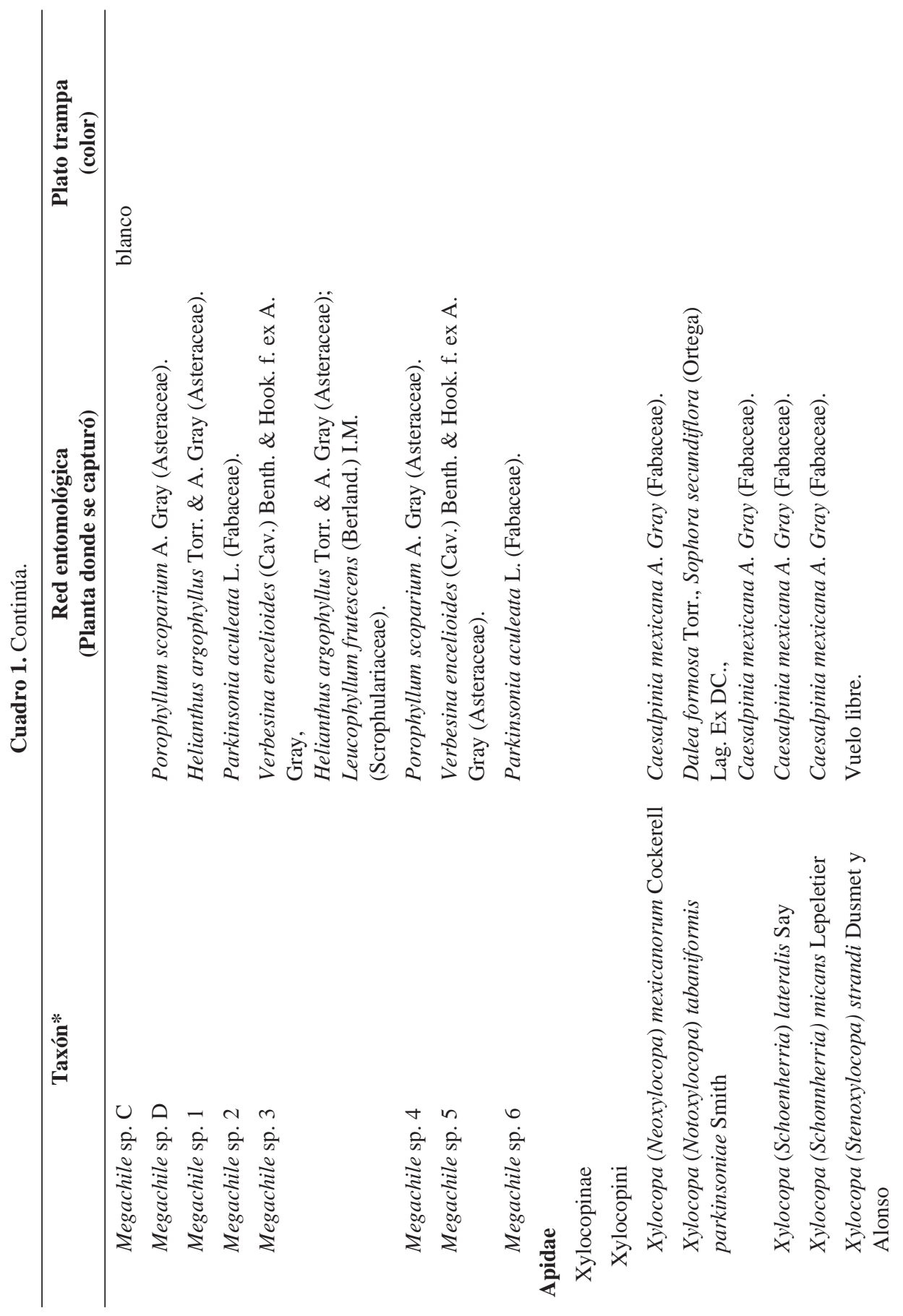


Acta Zool. Mex. (n.s.) 30(3) (2014)

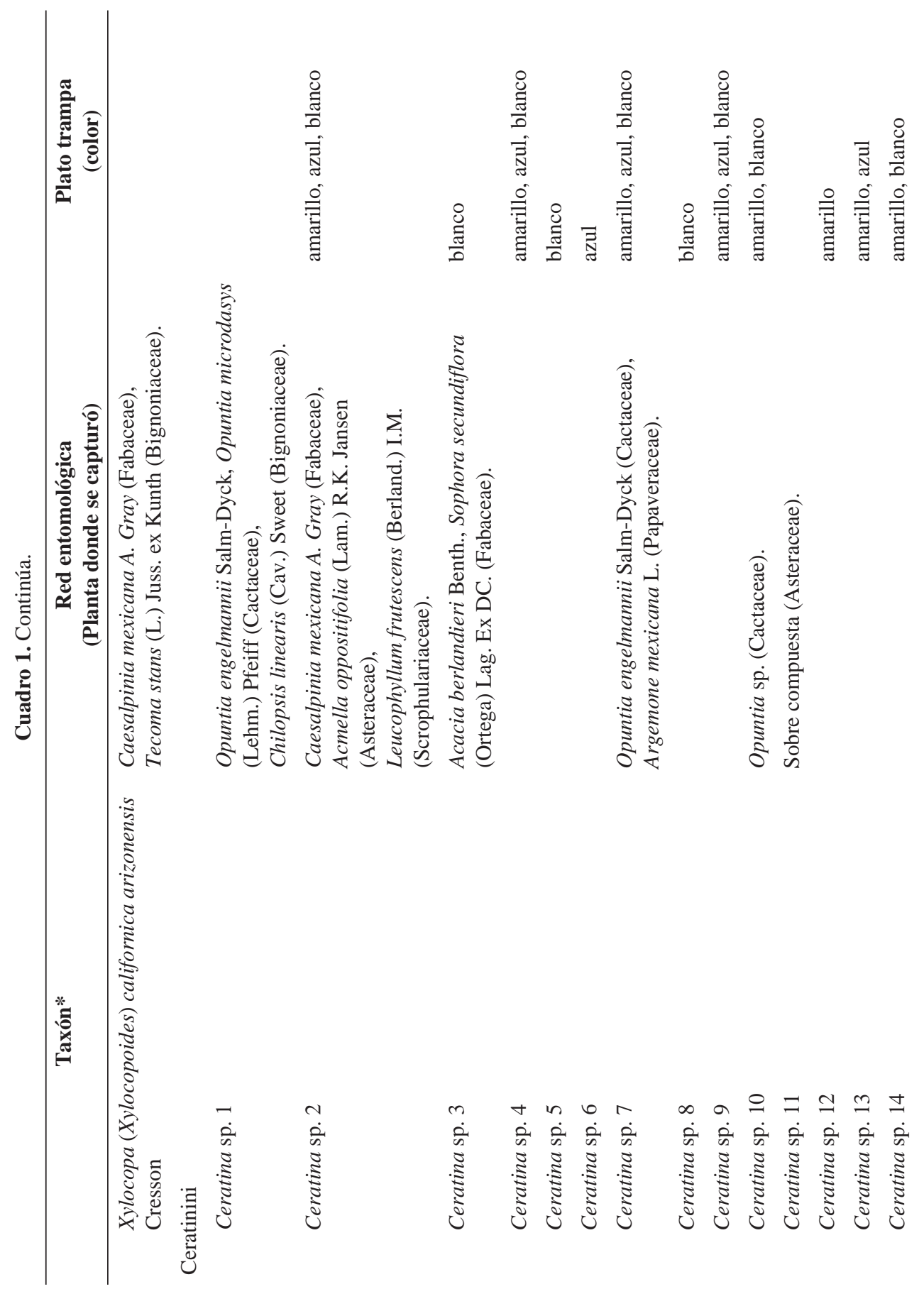




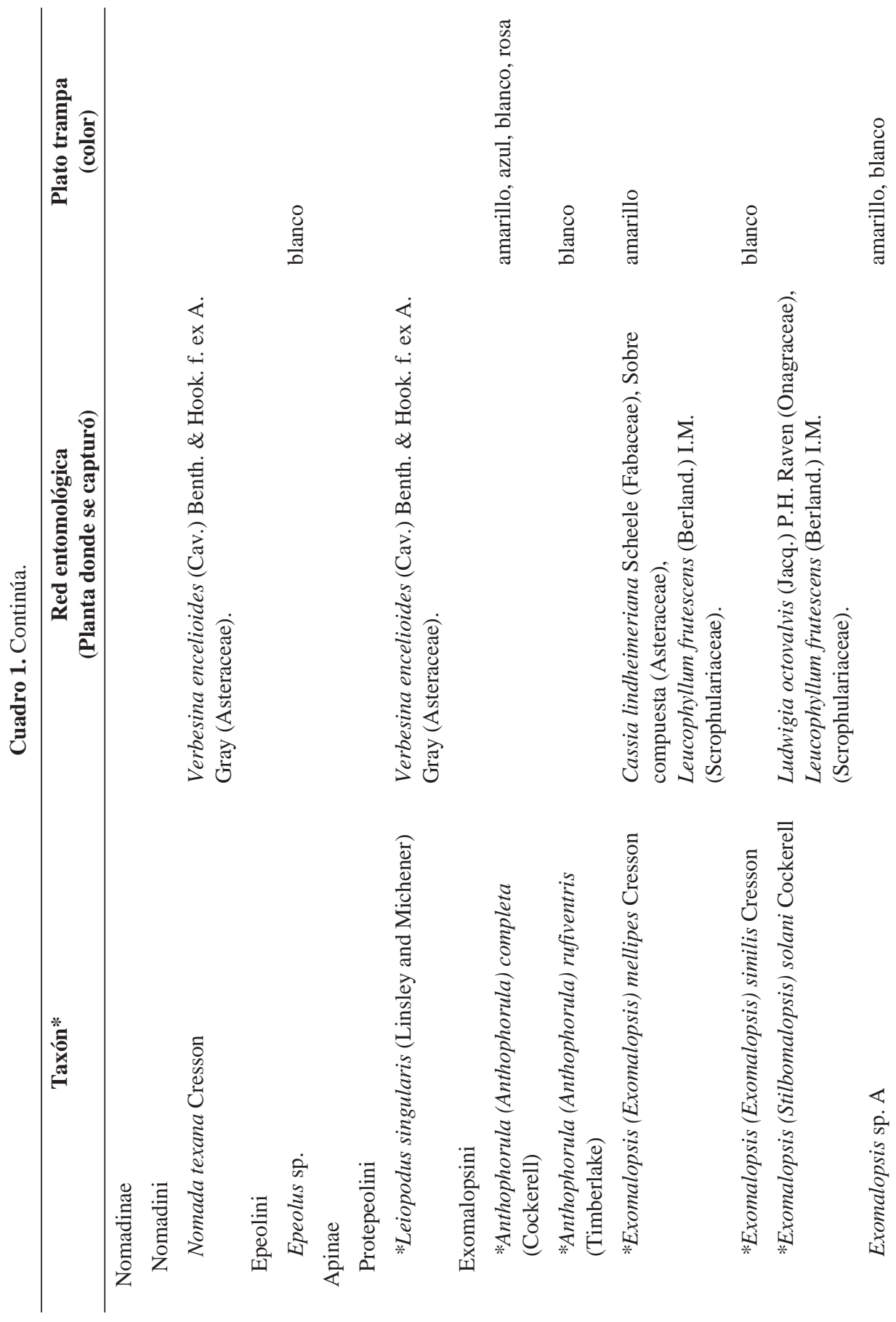


Acta Zool. Mex. (n.s.) 30(3) (2014)

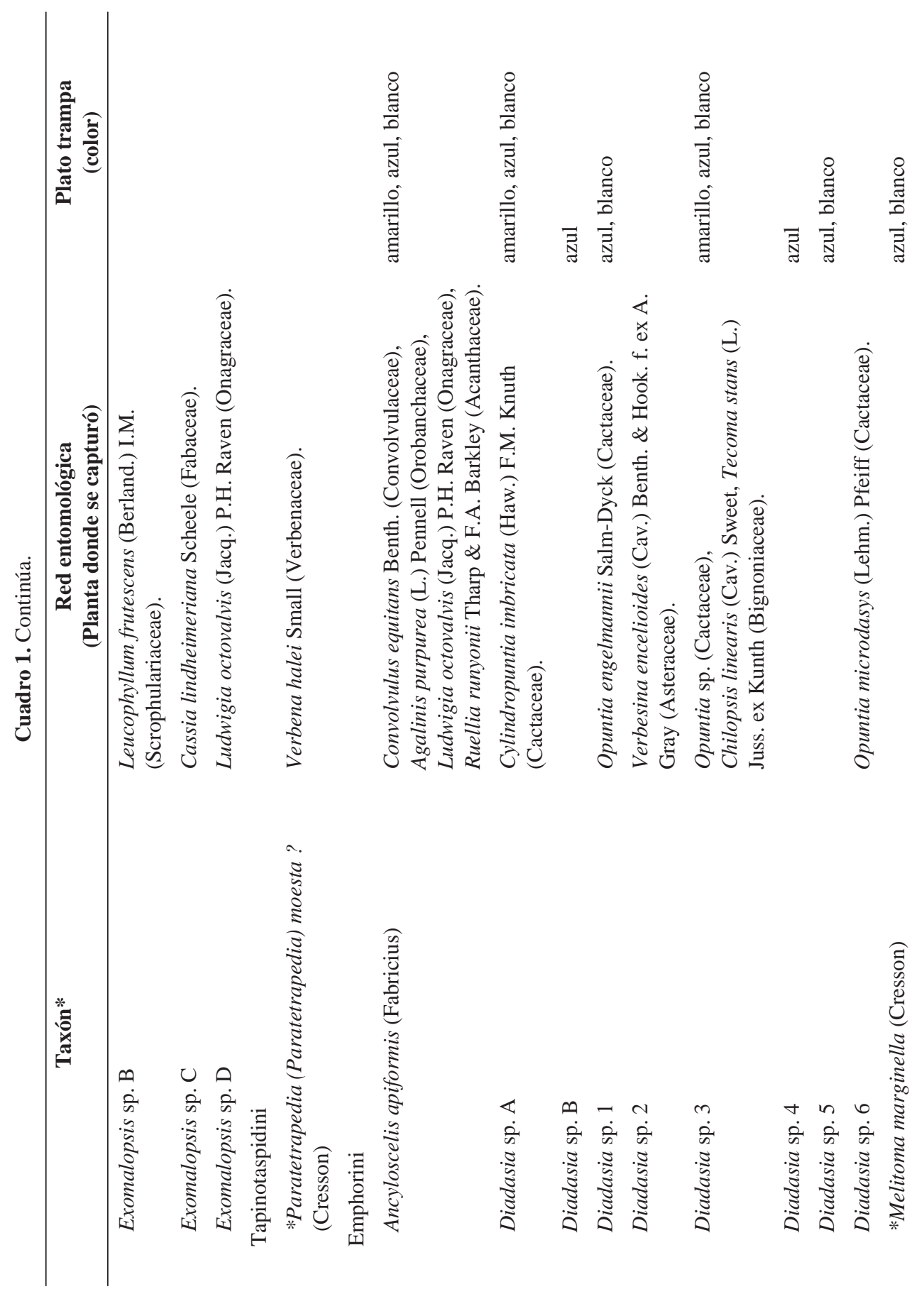




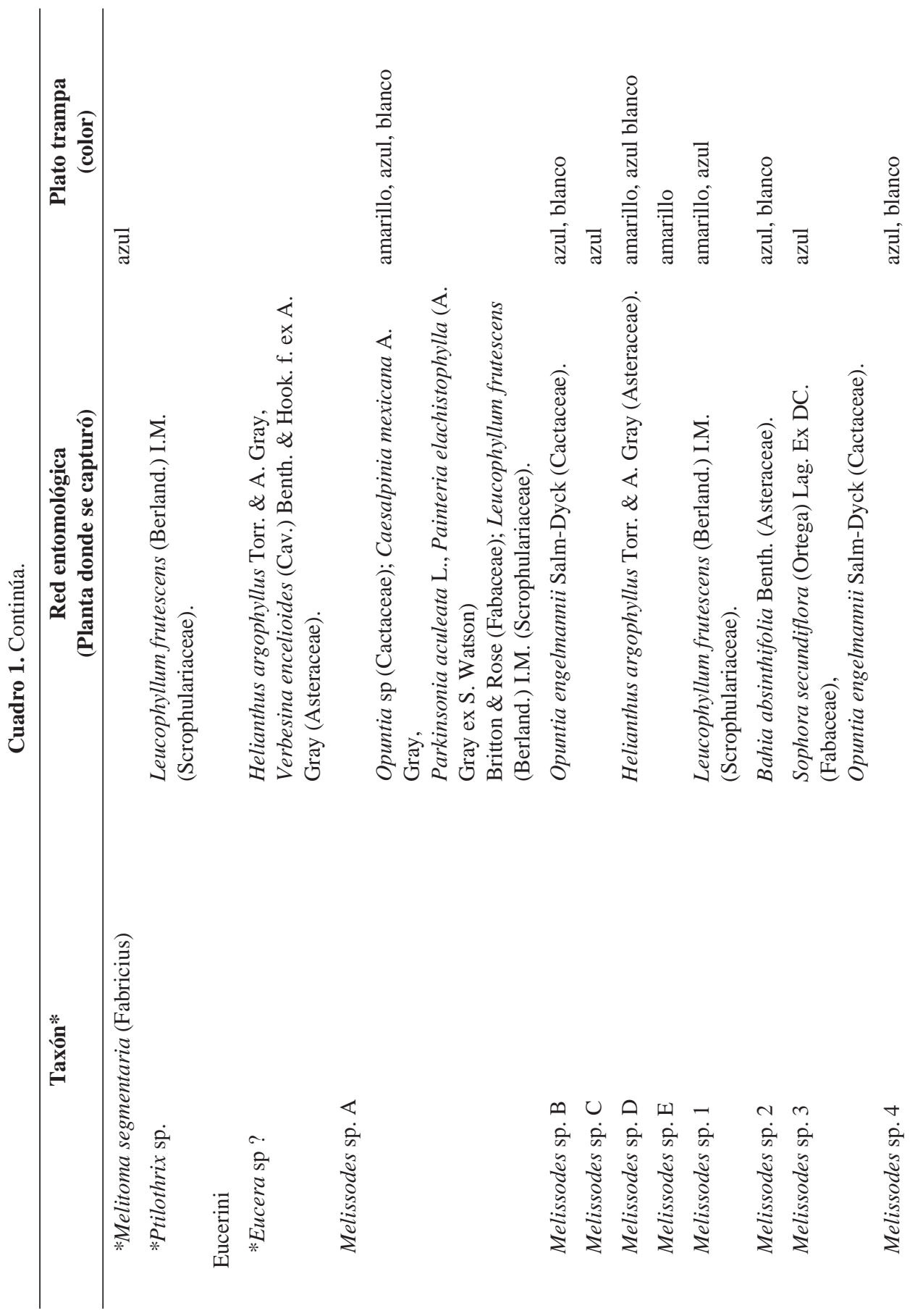




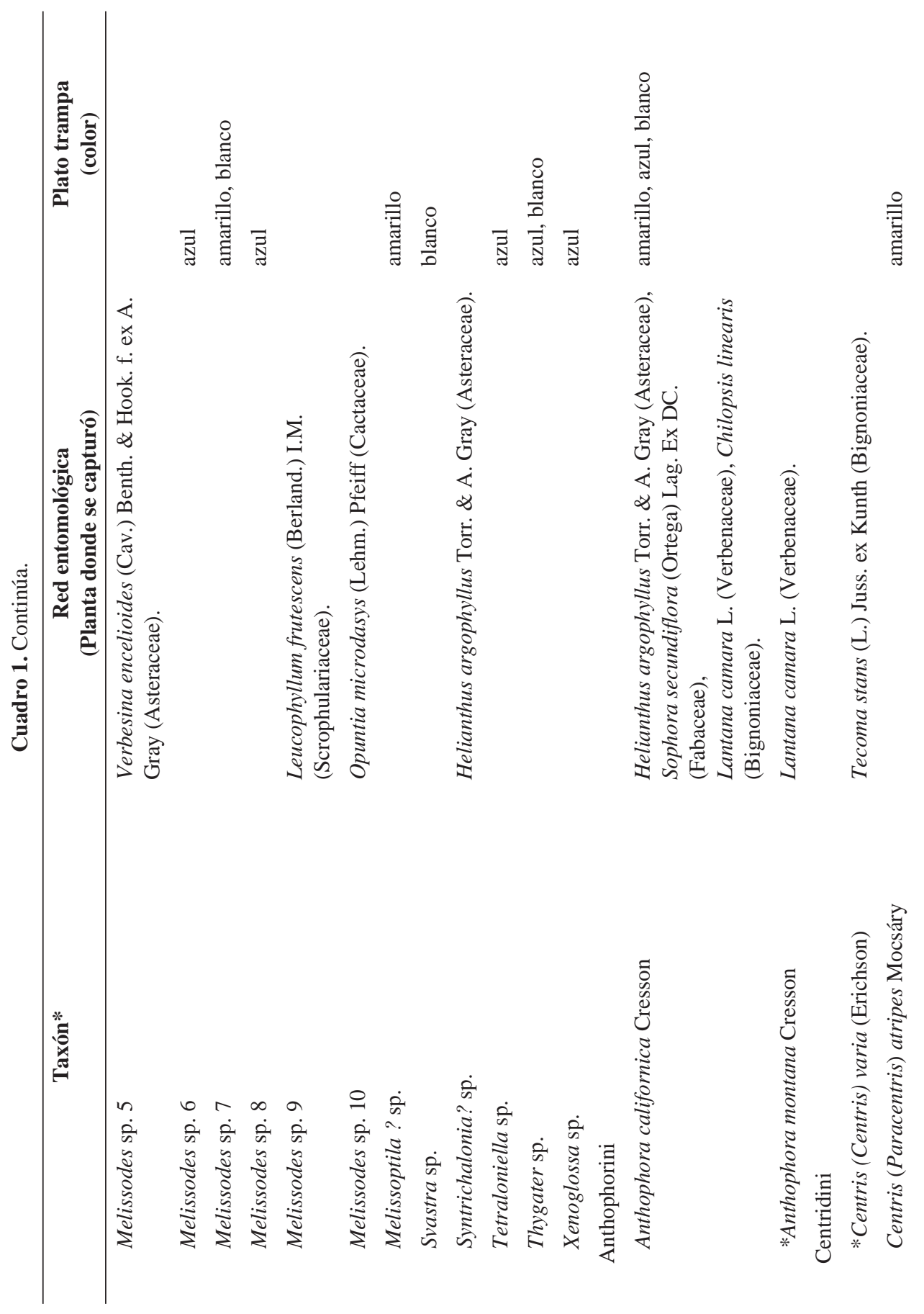




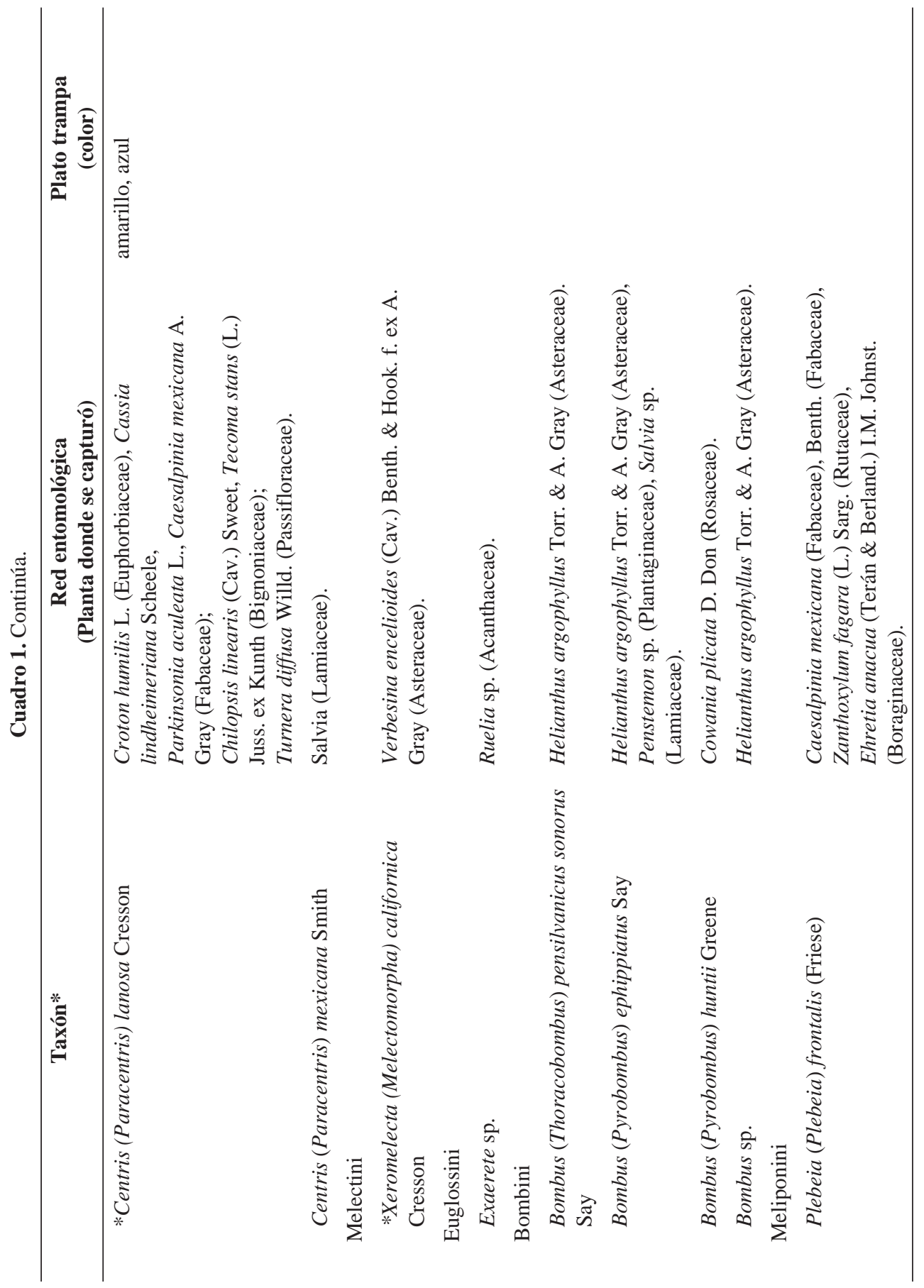




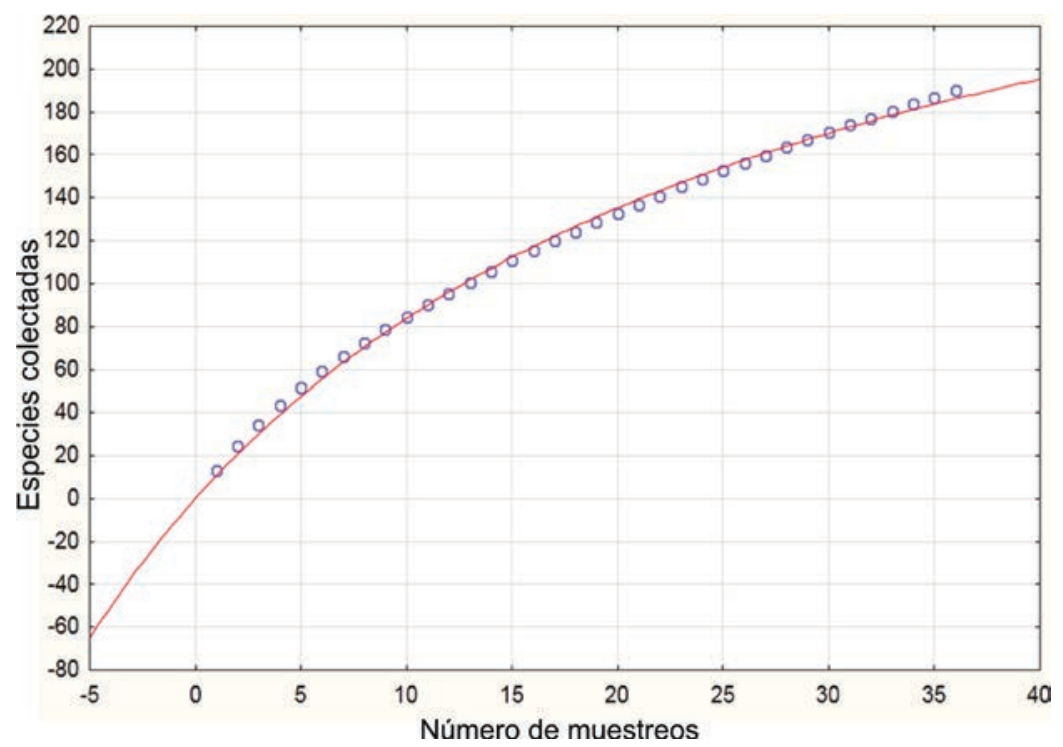

Figura 3. Curva de acumulación de especies de abejas nativas para el inventario faunístico realizado mediante el uso de red entomológica y platos trampa en el estado de Nuevo León, México usando EstimateS (Colwell, 2009). Se ajustó la curva a la ecuación de Clench $\left(\mathrm{S}_{\mathrm{col}}=191, \mathrm{R}^{2}=0.997, \mathrm{a} / \mathrm{b}=\right.$ 354). Los círculos representan los muestreos realizados y la línea continua representa la función de Clench.

Cuadro 2 se puede apreciar que en los platos trampa (aquí se incluye la información de todos los colores usados) se obtuvo una mayor abundancia $(\mathrm{N}=3,367)$ y riqueza de especies ( $\mathrm{S}=121)$; mientras que de acuerdo a los índices de diversidad, la red entomológica generó los más altos valores al aplicar el índice de Margalef, Simpson y Shannon $(\mathrm{DMg}=18.063$, Ds= 37.292 y H'= 4.138). Al realizar la prueba T de Student a ambos métodos de muestreo, se encontró que si existe una diferencia estadísticamente significativa entre ellos ya que el valor de P (0.001103) fue menor a 0.05. Por otra parte, al estimar el Índice de Jaccard se obtuvo un valor de $\mathrm{Ij}=0.273$, lo que

Cuadro 2. Abundancia, riqueza e índices de diversidad aplicados a los métodos de muestreo empleados para la captura de abejas nativas.

\begin{tabular}{|c|c|c|c|c|c|}
\hline \multirow[t]{2}{*}{ Tipo de muestreo } & \multirow{2}{*}{$\begin{array}{l}\text { Abundancia } \\
\text { (N) }\end{array}$} & \multirow{2}{*}{$\begin{array}{l}\text { Riqueza de sp } \\
\text { (S) }\end{array}$} & \multicolumn{3}{|c|}{ Diversidad } \\
\hline & & & $\begin{array}{c}\text { Margalef } \\
\text { (DMg) }\end{array}$ & $\begin{array}{l}\text { Simpson } \\
\text { (Ds) }\end{array}$ & $\begin{array}{c}\text { Shannon } \\
\left(\mathbf{H}^{\prime}\right)\end{array}$ \\
\hline Plato trampa & 3367 & 121 & 14.775 & 9.813 & 3.043 \\
\hline Red entomológica & 582 & 116 & 18.063 & 37.292 & 4.138 \\
\hline
\end{tabular}


nos indicó que la semejanza de las especies obtenidas entre la red entomológica y los platos trampa es baja.

Efectividad del color de los platos trampa. La abundancia de ejemplares por familia respecto a los colores utilizados con el método de platos trampa (PT), indicó que el color amarillo capturó el mayor número de ejemplares $(1,319)$, siendo la familia Halictidae la mejor representada. En contraste en los PT rosa se registró la menor cantidad (44 individuos). Se observa también que los PT azul y blanco capturaron un número similar de ejemplares para las familias Apidae y Andrenidae (Cuadro 3). Al analizar los resultados con ANOVA se encontró como valor de F (5.7249), siendo mayor al valor crítico de F (2.7482), por lo que existe una diferencia estadísticamente significativa entre los distintos colores empleados.

Ahora bien, los resultados con respecto a los colores de trampa usados se aprecian en el Cuadro 4, haciendo resaltar que en el amarillo se colectó una mayor abundancia $(\mathrm{N}=1319)$, riqueza $(\mathrm{S}=76)$ y diversidad de Margalef ( $\mathrm{DMg}=10.439)$; sin embargo, al comparar los resultados de los índices de Simpson y Shannon, el color azul presenta los más altos valores (Ds= 12.111 y H’= 3.105).

Los resultados arrojados por el Índice de Jaccard expuestos en el Cuadro 5, nos muestran que las trampas blancas y amarillas son las de mayor semejanza en cuanto

Cuadro 3. Abundancia de abejas nativas registrada con los diferentes colores utilizados en el método de platos trampa.

\begin{tabular}{|c|c|c|c|c|c|}
\hline \multirow{2}{*}{$\begin{array}{c}\text { Familia de } \\
\text { Apoidea }\end{array}$} & \multicolumn{4}{|c|}{ Color de los platos trampa } & \multirow[t]{2}{*}{ Total } \\
\hline & amarilla & blanca & azul & rosa & \\
\hline Colletidae & 1 & 0 & 1 & 0 & 2 \\
\hline Andrenidae & 92 & 135 & 138 & 3 & 368 \\
\hline Halictidae & 994 & 662 & 475 & 22 & 2153 \\
\hline Megachilidae & 23 & 32 & 16 & 3 & 74 \\
\hline Apidae & 209 & 266 & 279 & 16 & 770 \\
\hline Totales & 1319 & 1095 & 909 & 44 & 3367 \\
\hline
\end{tabular}

Cuadro 4. Abundancia, riqueza e índices de diversidad aplicados a los diferentes colores usados en el método de los platos trampa para la captura de abejas nativas.

\begin{tabular}{lccccc}
\hline $\begin{array}{c}\text { Color de } \\
\text { trampa }\end{array}$ & $\begin{array}{c}\text { Abundancia } \\
(\mathbf{N})\end{array}$ & $\begin{array}{c}\text { Riqueza de sp } \\
\text { (S) }\end{array}$ & $\begin{array}{c}\text { Margalef } \\
\text { (DMg) }\end{array}$ & $\begin{array}{c}\text { Simpson } \\
(\mathbf{D s})\end{array}$ & $\begin{array}{c}\text { Shannon } \\
\left(\mathbf{H}^{\prime}\right)\end{array}$ \\
\hline Amarillo & 1319 & 76 & 10.439 & 7.281 & 2.824 \\
Blanco & 1095 & 72 & 10.145 & 9.103 & 2.930 \\
Azul & 909 & 72 & 10.422 & 12.111 & 3.105 \\
Rosa & 44 & 18 & 4.492 & 5.29 & 2.135 \\
\hline
\end{tabular}


Cuadro 5. Resultados obtenidos del Coeficiente de Similitud de Jaccard aplicado entre los colores usados en el método de platos trampa.

\begin{tabular}{lcccc}
\hline Color de trampa & PT amarilla & PT azul & PT blanca & PT rosa \\
\hline Amarillo & & & & \\
Azul & 0.42 & & \\
Blanco & 0.48 & 0.47 & 0.16 & \\
Rosa & 0.17 & 0.16 & \\
\hline
\end{tabular}

a especies compartidas con $\mathrm{Ij}=0.48$, seguidas por las blancas y azules con $\mathrm{Ij}=0.47$; mientras que las trampas rosas comparadas con los demás colores fueron las menos similares.

Red Entomológica y plantas visitadas por abejas. El muestreo se realizó en plantas en floración, de las que se identificaron 21 familias que fueron visitadas por abejas, entre las que destacaron por la abundancia de éstas fueron Scrophulariaceae $(\mathrm{N}=$ 158), Asteraceae y Fabaceae (ambas con $\mathrm{N}=114$ ). Respecto a la riqueza de abejas, las familias de plantas que presentaron mayor número de especies fueron Asteraceae $(\mathrm{S}=36)$, Fabaceae $(\mathrm{S}=33)$, Cactaceae $(\mathrm{S}=27)$ y Scrophulariaceae $(\mathrm{S}=25)$. Respecto a los distintos índices de diversidad aplicados, Asteraceae presentó los valores más altos $\left(\mathrm{DMg}=7.391\right.$, Ds $=18.672$ y $\left.\mathrm{H}^{\prime}=3.201\right)$, seguido de Fabaceae $(\mathrm{DMg}=$ 6.756, Ds $=13.709$ y H'= 3.008) y Cactaceae $\left(\mathrm{DMg}=6.162\right.$, Ds $=13.844 \mathrm{y} \mathrm{H}^{\prime}=$ 2.947); mientras que los más bajos se observaron en Convolvulaceae, Euphorbiaceae, Malvaceae, Papaveraceae, Plantaginaceae, Passifloraceae y Zygophylaceae (Ds = 1, DMg y $\mathrm{H}^{\prime}=0$ ) (Cuadro 6).

Las familias botánicas donde se capturaron la mayoría de las familias de abejas fueron Asteraceae, Cactaceae, Fabaceae, Rosaceae y Scrophulariaceae; mientras que en las que se recolectaron abejas de sólo una familia fueron: Malvaceae con Andrenidae; Acanthaceae, Convolvulaceae, Euphorbiaceae, Lamiaceae, Papaveraceae, Plantaginaceae y Passifloraceae con Apidae; Brassicaceae y Zygophyllaceae con Halictidae. Por otra parte, las abejas de mayor ocurrencia en las familias de plantas fueron Apidae (en 17) y Halictidae (en 13); al contrario de Colletidae que se capturó únicamente en dos (Cuadro 7).

Se realizó una comparación entre las familias de plantas usando los resultados del índice de Jaccard, pero éste se aplicó únicamente a las que presentaron la mayor riqueza de especies tal y como se aprecian en el Cuadro 8, de las cuales Asteraceae y Fabaceae $(\mathrm{Ij}=0.115)$, seguidas de Fabaceae y Scrophulariaceae $(\mathrm{Ij}=0.108)$, son las más similares al compartir más especies que el resto; mientras que Asteraceae y Cactaceae son las más discrepantes $(\mathrm{Ij}=0.034)$. 
Cuadro 6. Índices de diversidad aplicados a las diferentes familias de plantas donde fueron capturadas abejas nativas mediante el uso de red entomológica en el estado de Nuevo León.

\begin{tabular}{lccccc}
\hline $\begin{array}{c}\text { Familias de } \\
\text { plantas }\end{array}$ & $\begin{array}{c}\text { Abundancia } \\
\text { (N) }\end{array}$ & $\begin{array}{c}\text { Riqueza de sp } \\
\text { (S) }\end{array}$ & $\begin{array}{c}\text { Margalef } \\
\text { (DMg) }\end{array}$ & $\begin{array}{c}\text { Simpson } \\
\text { (Ds) }\end{array}$ & $\begin{array}{c}\text { Shannon } \\
\left(\mathbf{H}^{\prime}\right)\end{array}$ \\
\hline Acanthaceae & 5 & 2 & 0.621 & 1.471 & 0.500 \\
Asteraceae & 114 & 36 & 7.391 & 18.672 & 3.201 \\
Bignoniaceae & 31 & 7 & 1.747 & 4.055 & 1.596 \\
Boraginaceae & 12 & 5 & 1.610 & 2.571 & 1.234 \\
Brassicaceae & 4 & 2 & 0.721 & 1 & 0.693 \\
Cactaceae & 68 & 27 & 6.162 & 13.844 & 2.947 \\
Convolvulaceae & 8 & 1 & 0 & 1 & 0 \\
Euphorbiaceae & 1 & 1 & 0 & 1 & 0 \\
Fabaceae & 114 & 33 & 6.756 & 13.709 & 3.008 \\
Lamiaceae & 4 & 3 & 1.443 & 2.667 & 1.040 \\
Malvaceae & 2 & 1 & 0 & 1 & 0 \\
Onagraceae & 5 & 5 & 2.485 & 5 & 1.609 \\
Papaveraceae & 1 & 1 & 0 & 1 & 0 \\
Plantaginaceae & 1 & 1 & 0 & 1 & 0 \\
Rhamnaceae & 3 & 2 & 0.910 & 1.8 & 0.636 \\
Rosaceae & 14 & 7 & 2.273 & 4.261 & 1.668 \\
Rutaceae & 12 & 2 & 0.402 & 1.180 & 0.287 \\
Scrophulariaceae & 158 & 25 & 4.741 & 5.557 & 2.353 \\
Passifloraceae & 1 & 1 & 0 & 1 & 0 \\
Verbenaceae & 10 & 6 & 2.171 & 5 & 1.696 \\
Zygophylaceae & 1 & 1 & 0 & 1 & 0 \\
\hline & & & & & 0 \\
\end{tabular}

\section{DISCUSIÓN}

El conocimiento sobre la diversidad de abejas mexicanas aún dista de ser completo y las estimaciones actuales podrían variar de manera importante si se realizaran muestreos más exhaustivos y en zonas poco exploradas como lo mencionaron Ayala et al. (1996) y un ejemplo de esto es que cuando se analiza a nivel de familia, las de mayor riqueza en México son Apidae y Andrenidae; no obstante, en el presente estudio, se encontró que Apidae, Halictidae e incluso Megachilidae son las que aportan más especies (Fig. 2), coincidiendo las dos primeras con lo reportado por Godínez-García et al. (2004) para el estado de Hidalgo, Fierros-López (2008) en el estado de Jalisco e Hinojosa-Díaz (2003) para el estado de Morelos.

El análisis sobre la calidad del muestreo realizado en este estudio mediante la curva de acumulación de especies y la ecuación de Clench muestra una pendiente que 
Cuadro 7. Familias de abejas nativas y familias de plantas donde fueron capturadas mediante el uso red entomológica en el estado de Nuevo León.

\begin{tabular}{|c|c|c|c|c|c|}
\hline Familia & Andrenidae & Apidae & Colletidae & Halictidae & Megachilidae \\
\hline Acanthaceae & & * & & & \\
\hline Asteraceae & $*$ & * & & $*$ & $*$ \\
\hline Bignoniaceae & & * & & $*$ & \\
\hline Boraginaceae & & * & & * & \\
\hline Brassicaceae & & & & $*$ & \\
\hline Cactaceae & $*$ & $*$ & & * & * \\
\hline Convolvulaceae & & * & & & \\
\hline Euphorbiaceae & & $*$ & & & \\
\hline Fabaceae & & $*$ & $*$ & $*$ & $*$ \\
\hline Lamiaceae & & $*$ & & & \\
\hline Malvaceae & $*$ & & & & \\
\hline Onagraceae & & $*$ & & $*$ & \\
\hline Papaveraceae & & $*$ & & & \\
\hline Plantaginaceae & & * & & & \\
\hline Ramnaceae & & & $*$ & $*$ & \\
\hline Rosaceae & * & * & & $*$ & $*$ \\
\hline Rutaceae & & $*$ & & $*$ & \\
\hline Scrophulariaceae & $*$ & $*$ & & $*$ & $*$ \\
\hline Passifloraceae & & * & & & \\
\hline Verbenaceae & & $*$ & & $*$ & \\
\hline Zygophyllaceae & & & & $*$ & \\
\hline Total & 5 & 17 & 2 & 13 & 5 \\
\hline
\end{tabular}

Cuadro 8. Resultados obtenidos del Coeficiente de Similitud de Jaccard, aplicado a las familias de plantas en las que se registró la mayor riqueza de especies de abejas.

\begin{tabular}{lcccc}
\hline \multicolumn{1}{c}{ Familia (\# sp) } & Asteraceae & Cactaceae & Fabaceae & Scrophulariaceae \\
\hline Asteraceae & - & & & \\
Cactaceae & 0.034 & - & & \\
Fabaceae & 0.115 & 0.054 & - & \\
Scrophulariaceae & 0.092 & 0.056 & 0.108 & - \\
\hline
\end{tabular}

indica que es necesario aumentar las fechas de muestreos para poder alcanzar la asíntota tal como lo mencionan Grundel et al. (2011), ya que al verificar el valor obtenido de a/b nos muestra que aún falta por colectar aproximadamente otras 163 especies. 
Por otra parte Jiménez \& Hortal (2003) mencionaron que la curva resultante con la ecuación de Clench para un inventario puede considerarse fiable a pesar de ser aún incompleto; además, si se reúnen las especies capturadas, más las registradas en literatura y las encontradas en las bases de datos de las colecciones revisadas, suman un total de 317 especies, cifra muy próxima al valor estimado de a/b, lo que nos acerca a tener un listado casi completo. Sin embargo, sería necesario más trabajo de campo o aumentar los sitios de muestreo (Grundel et al. 2011) para obtener los ejemplares.

Con respecto a los platos trampa, los resultados mostraron que ciertos colores capturan más abejas que otros (PT amarillos: 1319, PT rosa: 44); también se observó variación entre las diferentes familias de abejas y los distintos colores de trampa, donde en los PT amarillos capturaron más Halictidae, los PT azules ligeramente más Andrenidae y Apidae, PT blancos más Megachilidae, mientras que Colletidae no se capturó en ninguna. Por lo anterior, es claro que el color influye en la eficiencia de captura tal y como concluyeron Campbell \& Hanula (2007) y Leong \& Thorp (1999), quienes además mencionaron que pude afectar a nivel de especie e incluso el sexo de los ejemplares.

Otra observación es que abejas de talla grande como abejorrros (Bombus spp.) y abejas carpinteras (Xylocopa spp.) no fueron capturadas bajo este método, coincidiendo con lo reportado por Aizen \& Feinsinger (1992), Oerteli et al. (2005) y Sarospataki \& Fazekas (1995) quienes mencionan que éstas pueden ser pobremente representadas en platos trampa; no obstante Dobson et al. (1999) y Goulson et al. (2001) argumentaron que se debió a que son voladoras más eficientes que las abejas de talla pequeña, lo que les permite acercarse con mayor cautela a las trampas y usar otras señales sensoriales antes de posarse.

Según Hudson (2013), el uso de platos trampa puede dar resultados sesgados, ya que sólo el 19\% de las abejas atraídas caen en ellas y de éstas existen géneros que son capturados con más frecuencia como Lasioglossum, lo que provoca que se sobreestime su abundancia; también se puede falsear la presencia de abejas especializadas como las euglosinas, ya que tampoco se encuentran en los resultados y por el contrario favorecer familias generalistas como Halictidae (Cane et al. 2000).

A pesar de las limitaciones que pudiera tener el uso de platos trampa, éstos pueden capturar una muestra representativa de la comunidad de abejas siempre y cuando se utilicen en grandes cantidades, en zonas amplias, con múltiples colores y por períodos de tiempo prolongados; además siguen siendo la mejor alternativa para estudios que comparan diferentes hábitats o se quiere ver la estacionalidad (Hudson 2013).

De las familias botánicas donde se utilizó la red entomológica, Asteraceae y Fabaceae registraron un mayor número de especies de abejas; esto pudo deberse a la abundancia y diversidad que tienen estas plantas en el estado (Villarreal \& Estrada 2008, Velazco 2009) y a que fueron las que se encontraron más frecuentemente en 
floración. Por lo anterior otras familias que no fueron registradas en este trabajo, no significa que no sean visitadas por abejas, sino más bien que no se tuvo la oportunidad de colectar en ellas por no presentar flores.

Se observó que la red entomológica es apropiada para abejas de talla grande, aunque la habilidad del colector es determinante sobre todo para aquellas que son muy rápidas como las megaquílidas. Por otra parte, resulta poco favorable para ciertos grupos como las parasíticas, al respecto Oerteli et al. (2005) argumentan que es debido a que no pasan mucho tiempo en las flores; también es difícil capturar ejemplares muy pequeños, ya que no se detectan fácilmente, además de las especies que frecuentan cactáceas o algunas fabáceas, ya que pudieran escapar por el problema que causa el que la red se atore entre las espinas.

Los valores obtenidos de especies capturadas con red entomológica, aunque presentaron una abundancia más baja, en los índices de diversidad (DMg, Ds y $\mathrm{H}^{\prime}$ ) se registraron los valores más altos comparado con los platos trampa, esto concuerda con lo encontrado por Grundel et al. (2011) y Wilson et al. (2008), quienes además sugieren que estos métodos pudieran ser complementarios y más efectivos al emplearse en el mismo estudio ya que usados simultáneamente resultan una excelente opción porque permiten capturar y conocer un mayor diversidad de abejas.

AGRADECIMIENTOS. Al Consejo Nacional de Ciencia y Tecnología (CONACYT) por la beca otorgada para la realización de esta investigación durante los estudios de posgrado. Al Consejo Estatal de Flora y Fauna Silvestre de Nuevo León, A. C (CEFFSNL) y al Dr. John L. Neff del Central Texas Melittological Institute por su invaluable apoyo en la determinación taxonómica de ejemplares. A Natusalud, A. C. por su apoyo con el quipo de transporte para las salidas al campo. A los revisores de este manuscrito por sus valiosos comentarios y sugerencias.

\section{LITERATURA CITADA}

Aizen, M. A. \& Feinsinger, P. 1992. Habitat fragmentation, native insect pollinators and feral honey bees in Argentine Chaco Serrano. Ecological Applications, 4: 378-392.

Alanís, F. G. J. 1996. Vegetación y flora de Nuevo León, una guía botánico-ecológica. Impresora Monterrey, S.A. de C.V. México. Pp. 1-20.

Allen-Wardell, G., Bernhardt, P., Bitner, R., Burquez, A., Buchmann, S., Cane, J., Cox, P. A., Dalton, V., Feinsinger, P., Ingram, M., Inouye, D., Jones, C. E., Kennedy, K., Kevan, P. G., Koopowitz, H., Medellin, R., Medellin-Morales, S., Nabhan, G. P., Pavlik, B., Tepedino, V., Torchio, P. \& Walker, S. 1998. The potential consequences of pollinator declines on the conservation of biodiversity and stability of food crop yields. Conservation Biology, 12: 8-17.

Arduser, M. 2009. Anthophora of ENA (east of 100 the meridian). [internet]. Disponible en el sitio de red: http://www.nbii.gov/images/uploaded/152986_1254340944699_ENA_Anthophora_key.pdf [Revisado el 30 de agosto de 2010].

Ascher, J \& Pickering, J. 2010. Discover Life's Bee species guide. [internet]. Disponible en el sitio de red: www.discoverlife.org/mp/20q?search=Agapostemon [Revisado el 18 de marzo de 2010]. 
Ayala, R., Griswold, T. L. \& Yanega, D. 1996. Apoidea (Hymenoptera). Pp. 423-464. In: Llorente B.J., García A.N. y Soriano E. (Eds.). Biodiversidad, Taxonomía y Biogeografía de Artrópodos de México. Hacia una síntesis de su conocimiento. UNAM-CONABIO, México.

Ayala, R. 1999. Revisión de las abejas sin aguijón de México (Hymenoptera: Apidae: Meliponini). Folia Entomologica Mexicana, 106: 1-123.

Campbell, J. W. \& Hanula, J. L. 2007. Efficiency of Malaise traps and colored pan traps for collecting flower visiting insects from three forested ecosystems. Journal Insect Conservancy, 11: 399-408.

Cane, J. H., Minckley, R. L. \& Kervin, L. J. 2000. Sampling Bees (Hymenoptera: Apiformes) for Pollinator Community Studies: Pitfalls of Pan-Trapping. Journal of the Kansas Entomological Society, 73: 225-231.

Colwell, R. 2009. EstimateS: Statistical estimation of species richness and shared species from samples. Version 8.2. [internet]. Disponible en el sitio de red: http://viceroy.eeb.uconn.edu/EstimateS. [Revisado el 2 de febrero de 2012].

Dafni, A., Kevan, P. G. \& Husband, B. C. 2005. Practical Pollination Biology. Enviroquest, Ltd. Canadá. Pp 401-418.

Dobson, H., Dobson, H. E. M., Danielson, E. M. \& Wesep, I. D. V. 1999. Pollen odor chemicals as modulators of bumble bee foraging on Rosa rugosa Thunb. (Rosaceae). Plant Species Biology, 14: 153-166.

Droege, S. (Comp). 2009. The Very Handy Manual: How to Catch and Identify Bees and Manage a Collection. USA. 71 pp. [internet]. Disponible en el sitio de red: http://bees.tennessee.edu/publications/HandyBeeManual.pdf [Revisado el 25 de Junio de 2009].

Droege, S., Tepedino, V. J., Lebuhn, G., Link, W., Minckley, R. L., Chen, Q. \& Conrad, C. 2009. Spatial patterns of bee captures in North American bowl trapping surveys. Insect Conservation and Diversity, 3: 15-23.

Estrada, L. C. M. 1992. Abejas Silvestres (Hymenoptera: Apoidea) de la Sierra del Tigre, Jalisco. Tesis (Licenciado en Biología). Facultad de Ciencias. Universidad de Guadalajara. 89 p.

Fierros-López, H. E. 2008. Estudio de Ordenamiento Ecológico Territorial de Jalisco, Diagnóstico de los subsistemas, subsistema natural, Medio biótico, Diagnóstico. Centro de Estudios en Zoología, CUCBA, de la Universidad de Guadalajara. [internet]. Disponible en el sitio de red: http://www. acude.udg.mx/jalisciencia/diagnostico/biotico/faunajalisco/abejas/anexo1.html [Revisado el 2 de agosto de 2010].

Godínez-García, L. M. 1991. Algunos aspectos de la fenología de las abejas silvestres (Hymenoptera: Apoidea) de San Gregorio, Guanajuato. Tesis (Licenciatura), Facultad de ciencias, UNAM. $50 \mathrm{p}$.

Godínez-García, L. M., Hinojosa D., I. \& Yáñez O., O. 2004. Melitofauna (Insecta: Hymenoptera) de algunos bosques mesófilos de montaña. Pp. 321-331. In: I. Luna, J. J. Morrone y D. Espinosa (eds.). Biodiversidad de la Sierra Madre Oriental. Las prensas de Ciencias. México.

Goulson, D., Chapman, J. W. \& Hughes, W. H. O. 2001. Discrimination of unrewarding flowers by different bee species; direct detection of rewards and use of repellent scent marks. Journal of Insect Behavior, 14: 669-678.

Grundel, R., Frohnapple, K. J., Jean, R. P. \& Pavlovic, N. B. 2011. Effectiveness of Bowl Trapping and Netting for Inventory of a Bee Community. Environmental Entomology, 40: 374380.

Hinojosa-Díaz, I. A. 1996. Estudio faunístico de las abejas silvestres (Hymenoptera: Apoidea) del Pedregal de San Angel, D. F. Tesis (Licenciatura), Facultad de Ciencias, UNAM. 51 p.

Hinojosa-Díaz, I. A. 2003. Abejas silvestres (Hymenoptera: Apoidea) del declive sur de la Sierra del Chichinautzin, Morelos, México. Folia Entomológica Mexicana, 42: 1-20. 
Hudson, J. 2013. Assessing the efficiency of pan traps for collecting bees (Hymenoptera:Apoidea). [internet]. Disponible en el sitio de red: http://www.caes.uga.edu/Applications/attachments/files/ J\%20Hudson\%20Ento.pdf [revisado el 11 de septiembre del 2013].

Hurd, P. D. 1955. The Carpenter Bees of California. Bulletin of the California Insect Survey, 4: 34-72.

Hurd, P. D. \& Michener, C. D. 1955. The Megachiline Bees of California (Hymenoptera: Megachilidae). Bulletin of the California Insect Survey, 3: 1-247.

INEGI. 1986. Síntesis geográfica del estado de Nuevo León. Secretaría de Programación y Presupuesto. México, D. F.

INEGI. 2005. Marco geoestadístico municipal derivado del II conteo de población y vivienda nacional. Escala 1:1,000,000. Aguascalientes, México.

Jiménez, V. A. \& Hortal ,J. 2003. Las curvas de acumulación de especies y la necesidad de evaluar la calidad de los inventarios biológicos. Revista Ibérica de Aracnología, 8: 151-161.

Kearns, C. A \& Inouye, D. W. 1993. Techniques for Pollination Biologists. University Press of Colorado. USA. Pp. 263-275.

Kevan, P. G. \& Phillips, T. P. 2001. The economic impacts of pollinator declines: an approach to assessing the consequences. Conservation Ecology, 5: 8.

Labougle, J. M. 1990. Bombus of Mexico and Central America (Hymenoptera, Apidae). Science Bulletin, 54: 35-73.

Leong, J. M. \& Thorp, R. W. 1999. Colour-coded sampling: the pan trap colour preferences of oligolectic and nonoligolectic bees associated with a vernal pool plant. Ecological Entomology, 24: 329-335.

McGinley, R. J. 1986. Studies of Halictinae (Apoidea: Halictidae), I: Revision of New World Lasioglossum Curtis. Smithsonian Contributions to Zoology, 429: 1-294.

Michener, C. D., McGlinley, R. J. \& Danforth, B. 1994. The Bee Genera of North and Central America (Hymenoptera: Apoidea). Smithsonian Institution Press. Washington y London. 209 pp.

Michener, C. D. 2007. The Bees of the World. The Johns Hopkins University Press, $2^{\circ}$ ed., USA. 992 pp.

Miranda F. \& Hernández X., E. 1963. Los tipos de vegetación de México y su clasificación. Boletín de la Sociedad Botánica de México, 28: 29-176.

Mitchell, T. B. 1960. Bees of the eastern United States. I. [Introduction, Andrenidae, Colletidae, Halictidae, Mellitidae]. Technical bulletin. North Carolina Agricultural Experiment Station, 141: 1-538.

Mitchell, T. B. 1962. Bees of the eastern United States. II. [Megachilidae, Anthophoridae, Apidae s.s.]. Technical bulletin. North Carolina Agricultural Experiment Station, 152: 1-557.

Oerteli, S., Müller, A. \& Dorn, S. 2005. Ecological and seasonal patterns in the diversity of a species-rich assemblage (Hymenoptera: Apoidea: Apiformes). European Journal of Entomology, 102: 53-63.

Oliveira, R. C., Menezes, C., Egea, S. A. E. \& Imperatriz, F. V. L. 2012. Trap-nest for stingless bees (Hymenoptera, Meliponini). Apidologie, 44: 29-37.

Potts, S. G., Kevan, P. G. \& Boone, J. W. 2005. Consevation in pollination: collecting, surveying and monitoring. Pp. 401-434. In: A. Dafni, P. G. Kevan \& B. C. Husband (Eds.). Practical Pollination Biology. Enviroquest, Ltd. Canada.

Quesada, A. M. 2013. Informe final del proyecto "Evaluación de los impactos del cambio climático en polinizadores y sus consecuencias potenciales en el sector agrícola en México”. [internet]. Disponible en el sitio de red: http://www.inecc.gob.mx/descargas/cclimatico/2010_polinizadores.pdf [revisado el 10 de septiembre del 2013].

Ramírez-Freire, L. 2008. La floración de cinco especies de cactáceas y sus insectos asociados en el área natural protegida “Sierra Corral de los Bandidos” municipio de García, Nuevo León. Tesis (Maestría). Facultad de Ciencias Biológicas, U.A.N.L. 135 pp. 
Roberts, R. B. 1972. Revision of the bee genus Agapostemon (Hymenoptera: Halictidae). The University of Kansas Science Bulletin, 49: 437-590.

Rojas, M. P. 1965. Generalidades sobre el estado de Nuevo León y datos acerca de su flora. Tesis (Doctorado). Universidad Nacional Autónoma de México. México. 124 pp.

Roubik, D. W., Villanueva, R., Cabrera, E. F. \& Colli, W. 1991. Abejas Nativas de la Reserva de la Biosfera de Sian Ka'an. Pp. 317-320. In: L. D. Navarro y J. G. Robinson (eds.). Diversidad biológica de la reserva de la biosfera de Sian Ka'an, Quintana Roo, México. CIQRO. México.

Ruiz, C. E., Kasparyan, D. R., Coronado, B. J. M., Myartseva, S. N., Trjapitzin, V. A., Hernández, A. S. G. \& García, J. J. 2010. Hymenópteros de la Reserva "El Cielo", Tamaulipas, México. Dugesiana, 17: 53-71.

Rzedowski J. 1978. Vegetación de México. Limusa. México. D. F., Pp. 97-110.

Sarospataki, M \& Fazekas, J. P. 1995. Ecological characteristics of bee communities on a sandy grassland. Tiscia, 29: 41-46.

StatSoft. 2012. STATISTICA (data analysis software system and computer program manual). Versión 10. StatSoft, Inc.

UNIBIO. 2011. CNIN/Abejas de México/Apodea. In Unidad de Informática para la Biodiversidad, Instituto de Biología, UNAM, México, D. F. http://unibio.unam.mx/minidigir/main.jsp?accion=sc\&co lecciones=CNIN; última consulta: 02.II.2011.

Velazco, M. C. G. 2009. Flora del estado de Nuevo León, México: Diversidad y Análisis Espacio- Temporal. Tesis (Doctorado). Facultad de Ciencias Biológicas, UANL. México. 299 p.

Vergara, C. H. \& Ayala, R. 2002. Diversity, phenology and biogeography of the bees (Hymenoptera: Apoidea) of Zapotitlan de las Salinas, Puebla, Mexico. Journal of the Kansas Entomological Society, 75: 16-30.

Villarreal, I. J. A. \& Estrada, E. C. 2008. Flora de Nuevo León. Listados Florísticos de México No. XXIV. Instituto de Biología, UNAM. México. 153 p.

Wilson, J. S., Griswold, T. \& Messinger, O. J. 2008. Sampling bee communities (Hymenoptera: Apiformes) in a desert landscape: are pan traps sufficient? Journal of the Kansas Entomological Society, 81: 288-300. 